\title{
Riemann-Hilbert Problems
}

\author{
Percy Deift
}

\begin{abstract}
These lectures introduce the method of nonlinear steepest descent for Riemann-Hilbert problems. This method finds use in studying asymptotics associated to a variety of special functions such as the Painlevé equations and orthogonal polynomials, in solving the inverse scattering problem for certain integrable systems, and in proving universality for certain classes of random matrix ensembles. These lectures highlight a few such applications.
\end{abstract}

\section{Contents}

Lecture 1 1

$\begin{array}{lr}\text { Lecture } 2 & 10\end{array}$

Lecture $3 \quad 20$

$\begin{array}{lr}\text { Lecture } 4 & 29\end{array}$

\section{Lecture 1}

These four lectures are an abridged version of 14 lectures that I gave at the Courant Institute on RHPs in 2015. These 14 lectures are freely available on the AMS website AMS Open Notes.

Basic references for RHPs are $[8,12,28]$. Basic references for complex function theory are $[19,23,24]$. Many more specific references will be given as the course proceeds.

Special functions are important because they provide explicitly solvable models for a vast array of phenomena in mathematics and physics. By "special functions" I mean Bessel functions, Airy functions, Legendre functions, and so on. If you have not yet met up with these functions, be assured, sooner or later, you surely will.

It works like this. Consider the Airy equation (see, e.g. [1,29])

$$
y^{\prime \prime}(x)=x y(x), \quad-\infty<x<\infty .
$$

Seek a solution of (1.1) in the form

$$
y(x)=\int_{\Sigma} e^{x s} f(s) d s
$$


for some functions $f(x)$ and some contours $\Sigma$ in the complex plane $\mathbb{C}$. We have

and

$$
y^{\prime \prime}(x)=\int_{\Sigma} s^{2} e^{x s} f(s) d s
$$

$$
\begin{aligned}
x y(x) & =\int_{\Sigma}\left(\frac{d}{d s} e^{x s}\right) f(s) d s \\
& =-\int e^{x s} f^{\prime}(s) d s
\end{aligned}
$$

provided we can drop the boundary terms. In order to solve (1.1) we need to have

$$
-f^{\prime}(s)=s^{2} f
$$

and so

$$
f(s)=\text { const. } e^{-\frac{1}{3} s^{3}} .
$$

Thus

$$
y(x)=\text { const. } \int_{\Sigma} e^{x s-\frac{1}{3} s^{3}} d s
$$

provides a solution of the Airy equation.

The particular choice

$$
\text { const. }=\frac{1}{2 \pi i}
$$

and $\Sigma$ in Figure 1.3 is known as Airy's integral $\mathrm{Ai}(\mathrm{x})$

$$
A i(x)=\frac{1}{2 \pi i} \int_{\Sigma} e^{x z-\frac{1}{3} z^{3}} d z .
$$

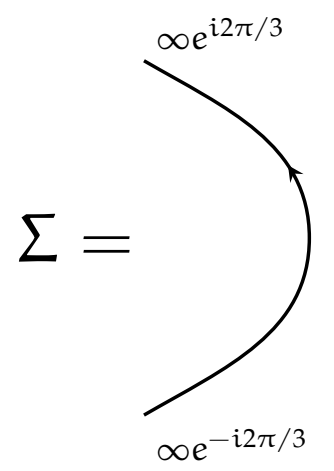

FIGURE 1.3. $\Sigma$ for Airy's integral.

Other contours provide other, independent solutions of Airy's equation, such as $\operatorname{Bi}(x)$ (see [1]). Now the basic fact of the matter is that the integral representation (1.2) for $A i(x)$ enables us, using the classical method of stationary phase/steepest descent, to compute the asymptotics of $A i(x)$ as $x \rightarrow+\infty$ and $-\infty$ with any desired 
accuracy. We find, in particular [1, p. 448], that for $\zeta=\frac{2}{3} x^{3 / 2}$

$$
\operatorname{Ai}(x) \sim \frac{1}{2 \sqrt{\pi}} x^{-\frac{1}{4}} e^{-\zeta} \sum_{k=0}^{\infty}(-1)^{k} c_{k} \zeta^{-k}
$$

as $x \rightarrow+\infty$, where

$$
\begin{aligned}
& c_{0}=1, \\
& c_{k}=\frac{\Gamma\left(3 k+\frac{1}{2}\right)}{54^{k} k ! \Gamma\left(k+\frac{1}{2}\right)}=\frac{(2 k+1)(2 k+3) \ldots(6 k-1)}{(216)^{k} k !}, \quad k \geqslant 1 .
\end{aligned}
$$

and that

$$
\begin{aligned}
\operatorname{Ai}(-x) \sim \frac{1}{\sqrt{\pi}} x^{-1 / 4} & \left(\sin \left(\zeta+\frac{\pi}{4}\right) \sum_{0}^{\infty}(-1)^{\mathrm{k}} \mathrm{c}_{2 \mathrm{k}} \zeta^{-2 \mathrm{k}}\right. \\
& \left.-\cos \left(\zeta+\frac{\pi}{4}\right) \sum_{0}^{\infty}(-1)^{\mathrm{k}} \mathrm{c}_{2 \mathrm{k}+1} \zeta^{-2 \mathrm{k}-1}\right)
\end{aligned}
$$

as $x \rightarrow+\infty$.

Such results for solutions of general $2^{\text {nd }}$ order equations are very rare. Formulae (1.4) and (1.5) solve the fundamental connection problem or scattering problem for solutions of the Airy equation. Thus, if we know that a solution $y(x)$ of the Airy equation behaves like

$$
y(x)=\frac{1}{2 \sqrt{\pi}} x^{-1 / 4} e^{-\zeta}\left(1-\frac{c_{1}}{\zeta}+\ldots\right)
$$

as $x \rightarrow+\infty$, then we know precisely how it behaves as $x \rightarrow-\infty$, and vice versa, by (1.4) (1.5), see Figure 1.6.

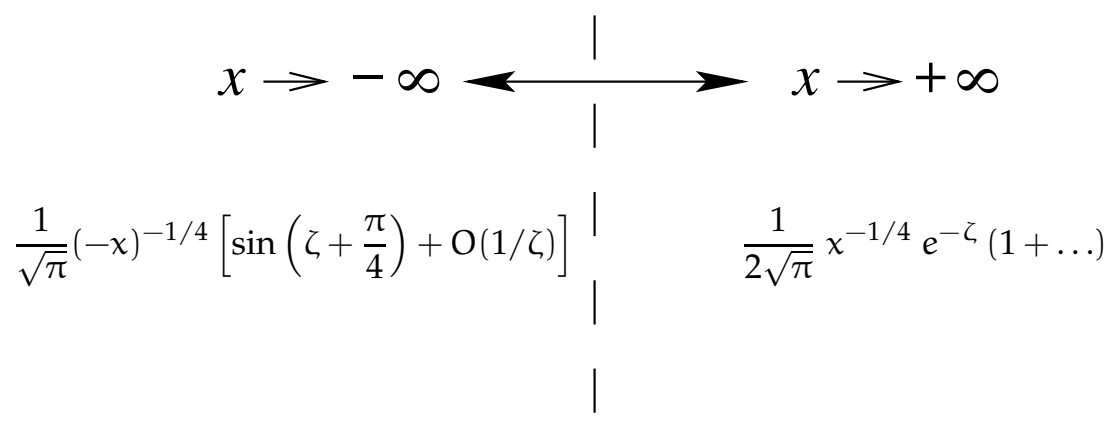

FIGURE 1.6. Asymptotics for Airy's integral.

Exercise 1.7. Use the classical steepest-descent method to verify (1.4) and (1.5). There are similar precise results for all the classical special functions. The diligent student should regard Abramowitz \& Stegun [1] as an exercise book for the steepest descent method - verify all the asymptotic formulae!

Now in recent years it has become clear that a new and extremely broad class of problems in mathematics, engineering and physics is described by a new class of special functions, the so-called Painlevé functions. There are six Painlevé equations 
and we will say more about them later on. Whereas the classical special functions, such as Airy functions, Bessel functions, etc. typically arise in linear (or linearized problems) such as acoustics or electromagnetism, the Painlevé equations arise in nonlinear problems, and they are now recognized as forming the core of modern special function theory. Here are some examples of how Painlevé equations arise:

Example 1.8. Consider solutions of the modified Korteweg-de Vries equation $(\mathrm{MKdV})$

$$
\begin{aligned}
& u_{t}-6 u^{2} u_{x}+u_{x x x}=0,-\infty<x<\infty, \quad t>0, \\
& u(x, 0)=u_{0}(x) \rightarrow 0 \text { as }|x| \rightarrow \infty .
\end{aligned}
$$

Then [16] as $t \rightarrow \infty$, in the region $|x| \leqslant c t^{1 / 3}, c<\infty$,

$$
u(x, t)=\frac{1}{(3 t)^{1 / 3}} \quad p\left(\frac{x}{(3 t)^{1 / 3}}\right)+O\left(\frac{1}{t^{2 / 3}}\right)
$$

where $\mathrm{p}(\mathrm{s})$ is a particular solution of the Painlevé II (PII) equation

$$
p^{\prime \prime}(s)=s p(s)+2 p^{3}(s) .
$$

Example 1.11. Let $\pi:=\left(\pi_{1} \pi_{2} \ldots \pi_{N}\right) \in S_{N}$ be a permutation of the numbers $1,2, \ldots, N$. We say that $\pi_{i_{1}}, \pi_{i_{2}}, \ldots \pi_{i_{k}}$ is an increasing subsequence of $\pi$ of length $k$ if

$$
i_{1}<i_{2}<\cdots<i_{k}
$$

and

$$
\pi_{i_{1}}<\pi_{i_{2}}<\cdots<\pi_{i_{k}} .
$$

Thus if $N=6$ and $\pi=(413265)$, then 125 and 136 are increasing subsequences of $\pi$ of length 3 . Let $\ell_{N}(\pi)$ denote the length of a longest increasing subsequence of $\pi$, e.g., for $N=6$ and $\pi$ as above, $\ell_{6}(\pi)=3$, which is the length of the longest increasing subsequences 125 and 136.

Now equip $S_{\mathrm{N}}$ with uniform measure. Thus

$$
\operatorname{Prob}\left(\ell_{\mathrm{N}} \leqslant \mathrm{n}\right)=\frac{\#\left\{\pi \in S_{N}: \ell_{N}(\pi) \leqslant n\right\}}{\mathrm{N} !} \text {. }
$$

Question. How does $\ell_{N}$ behave statistically as $N, n \rightarrow \infty$ ?

Theorem 1.12 ([2]). Center and scale $\ell_{N}$ as follows:

then

$$
\ell_{N} \rightarrow X_{N}=\frac{\ell_{N}-2 \sqrt{N}}{N^{1 / 6}}
$$

$$
\lim _{N \rightarrow \infty} \operatorname{Prob}\left(X_{N} \leqslant x\right)=e^{-\int_{x}^{\infty}(s-x) u^{2}(s) d s}
$$

where $\mathrm{u}(\mathrm{s})$ is the (unique) solution of Painlevé II (the so-called Hastings-McLeod solution) normalized such that

$$
\mathrm{u}(\mathrm{s}) \sim \mathrm{Ai}(\mathrm{s}) \text { as } \mathrm{s} \rightarrow+\infty .
$$

The distribution on the right in Theorem 1.12 is the famous Tracy-Widom distribution for the largest eigenvalue of a GUE matrix in the edge scaling limit. 
Theorem 1 is one of a very large number of probabilistic problems in combinatorics and related areas, whose solution is expressed in terms of Random Matrix Theory (RMT) via Painlevé functions (see, e.g., [3]).

The key question is the following: Can we describe the solutions of the Painlevé equations as precisely as we can describe the solutions of the classical special functions such as Airy, Bessel, ... ? In particular, can we describe the solutions of the Painleve equations asymptotically with arbitrary precision and solve the connection/scattering problem as in (1.4) and (1.5) for the Airy equation (or any other of the classical special functions):

$$
\text { known behavior as } x \rightarrow+\infty \quad \Rightarrow \quad \text { known behavior as } x \rightarrow-\infty
$$

and vice versa.

As we have indicated, at the technical level, connection formulae such as (1.4) and (1.5) can be obtained because of the existence of an integral representation such as (1.2) for the solution. Once we have such a representation the asymptotic behavior is obtained by applying the (classical) steepest descent method to the integral. There are, however, no known integral representations for solutions of the Painlevé equations and we are led to the following questions:

Question 1: Is there an analog of an integral representation for solutions of the Painlevé equations?

Question 2: Is there an analog of the classical steepest descent method which will enable us to extract precise asymptotic information about solutions of the Painlevé equations from this analog representation?

The answer to both questions is yes: In place of an integral representation such as (1.2), we have a Riemann-Hilbert Problem (RHP), and in place of the classical steepest descent method we have the nonlinear (or non-commutative) steepest descent method for RHPs (introduced by P. Deift and X. Zhou [16]).

So what is a RHP? Let $\Sigma$ be an oriented contour in the plane, see Figure 1.13.

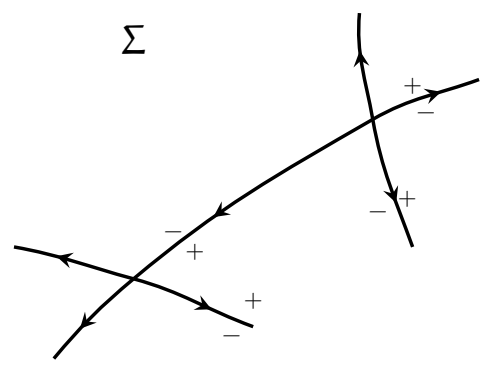

FIGURE 1.13. An oriented contour in the plane.

By convention, if we move along an arc in $\Sigma$ in the direction of the orientation, the $( \pm)$-sides lie on the left (resp. right). Let $v: \Sigma \rightarrow \mathrm{GL}(k, \mathbb{C})$, the jump matrix, be an invertible $\mathrm{k} \times \mathrm{k}$ matrix function defined on $\Sigma$ with

$$
v, v^{-1} \in \mathrm{L}^{\infty}(\Sigma)
$$


We say that an $n \times k$ matrix function $m(z)$ is a solution of the RHP $(\Sigma, v)$ if

If, in addition, $\mathrm{n}=\mathrm{k}$ and

$$
\begin{aligned}
& \mathrm{m}(z) \text { is analytic in } \mathbb{C} / \Sigma, \\
& \mathrm{m}_{+}(z)=\mathrm{m}_{-}(z) v(z), z \in \Sigma, \\
& \text { where } \mathrm{m}_{ \pm}(z)=\lim _{z^{\prime} \rightarrow z_{ \pm}} \mathrm{m}\left(z^{\prime}\right) .
\end{aligned}
$$

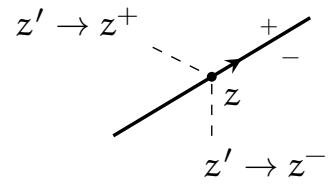

$$
\mathrm{m}(z) \rightarrow \mathrm{I}_{\mathrm{k}} \quad \text { as } \quad z \rightarrow \infty,
$$

we say that $\mathrm{m}(z)$ solves the normalized $\operatorname{RHP}(\Sigma, v)$.

RHPs involve a lot of technical issues. In particular

- How smooth should $\Sigma$ be?

- What measure theory/function spaces are suitable for RHPs?

- What happens at points of self intersection (see Figure 1.14)?

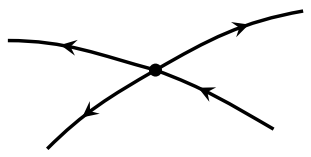

FIGURE 1.14. A point of self intersection.

- In what sense are the limits $m_{ \pm}(z)$ achieved?

- In the case $n=k$, in what sense is the limit $m(z) \rightarrow I_{k}$ achieved?

- Does an $n \times k$ solution exist?

- In the normalized case, is the solution unique?

And most importantly

- at the analytical level, what kind of problem is a RHP? As we will see, the problem reduces to the analysis of singular integral equations on $\Sigma$.

There is not enough time in these 4 lectures to address all these issues systematically. Rather we will address specific issues as they arise.

As an example of how things work, we now show how PII is related to a RHP (see, e.g. [22]). Let $\Sigma$ denote the union of six rays

$$
\Sigma_{\mathrm{k}}=e^{i(k-1) \pi / 3} \rho, \quad \rho>0, \quad 1 \leqslant k \leqslant 6
$$

oriented outwards. Let $p, q, r$ be complex numbers satisfying the relation

$$
p+q+r+p q r=0 .
$$

Let $v(z), z \in \Sigma$, be constant on each ray as indicated in Figure 1.16 and for fixed $x \in \mathbb{C}$ set

$$
v_{x}(z)=\left(\begin{array}{cc}
e^{-i \theta} & 0 \\
0 & e^{i \theta}
\end{array}\right) v(z)\left(\begin{array}{cc}
e^{i \theta} & 0 \\
0 & e^{-i \theta}
\end{array}\right), \quad z \in \Sigma
$$

where

$$
\theta=\theta_{\chi}(z)=\frac{4}{3} z^{3}+x z
$$


Thus for $z \in \Sigma_{3}$

$$
v_{x}(z)=\left(\begin{array}{cc}
1 & r e^{-2 i \theta} \\
0 & 1
\end{array}\right)
$$

and so on.

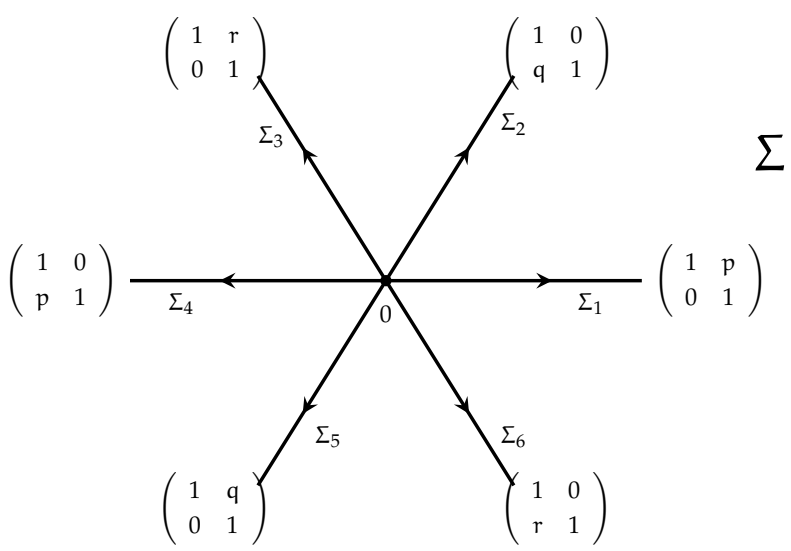

FIGURE 1.16. Six rays oriented outwards.

For fixed $x$, let $m_{x}(z)$ be the $2 \times 2$ matrix solution of the normalized $\operatorname{RHP}\left(\Sigma, v_{x}\right)$. Then

$$
u(x)=2 i\left(m_{1}(x)\right)_{12}
$$

is a solution of the PII equation where

$$
\mathrm{m}_{\mathrm{x}}(z)=\mathrm{I}+\frac{\mathrm{m}_{1}(\mathrm{x})}{z}+\mathrm{O}\left(\frac{1}{z^{2}}\right)
$$

as $z \rightarrow \infty$. (This result is due to Jimbo and Miwa [27], and independently to Flaschka and Newell [20].) The asymptotic behavior of $u(x)$ as $x \rightarrow \infty$ is then obtained from the RHP $\left(\Sigma, v_{x}\right)$ by the nonlinear steepest descent method.

In the classical steepest descent method for integrals such as (1.2) above, the contour $\Sigma$ is deformed so that the integral passes through a stationary phase point where the integrand is maximal and the main contribution to the integral then comes from a neighborhood of this point. The nonlinear (or non-commutative) steepest descent method for RHPs involves the same basic ideas as in the classical scalar case in that one deforms the RHP, $\Sigma \rightarrow \Sigma^{\prime}$, in such a way that the exponential terms (see e.g. $e^{2 i \theta}$ above) in the RHP have maximal modulus at points of the deformed contour $\Sigma^{\prime}$. The situation is far more complicated than the scalar integral case, however, as the problem involves matrices that do not commute. In addition, terms of the form $e^{-2 i \theta}$ also appear in the problem and must be separated algebraically from terms involving $e^{2 i \theta}$, so that in the end the terms involving $e^{2 i \theta}$ and $e^{-2 i \theta}$ both have maximal modulus along $\Sigma^{\prime}$ (see [16-18]). A simple example of the nonlinear steepest descent method is given at the end of Lecture 4. 
One finds, in particular, ([18], and also [22,25]) the following:

Let $-1<q<1, p=-q, r=0$. Then as $x \rightarrow-\infty$,

$$
u(x)=\frac{\sqrt{2 v}}{(-x)^{1 / 4}} \cos \left(\frac{2}{3}(-x)^{3 / 2}-\frac{3}{2} v \log (-x)+\phi\right)+O\left(\frac{\log (-x)}{(-x)^{5 / 4}}\right)
$$

where

$$
v=v(q)=-\frac{1}{2 \pi} \log \left(1-q^{2}\right)
$$

and

$$
\phi=-3 \vee \log 2+\arg \Gamma(\mathfrak{i} v)+\frac{\pi}{2} \operatorname{sgn}(\mathfrak{q})-\frac{\pi}{4}
$$

As $x \rightarrow+\infty$

$$
u(x)=q A i(x)+O\left(\frac{e^{-4 / 3 x^{3 / 2}}}{x^{1 / 4}}\right) .
$$

These asymptotics should be compared with (1.4), (1.5) for the Airy function. Note from (1.4) that as $x \rightarrow+\infty$

$$
A i(x) \sim x^{-1 / 4} e^{-2 / 3 x^{3 / 2}} .
$$

Also observe that PII

$$
u^{\prime \prime}(x)=x u(x)+2 u^{3}(x)
$$

is a clearly a nonlinearization of the Airy equation

$$
u^{\prime \prime}(x)=x u(x)
$$

and so we expect similar solutions when the nonlinear term $2 u^{3}(x)$ is small.

Also note that (1.17) and (1.18) solve the connection problem for PII. If we know the behavior of the solutions $u(x)$ of PII as $x \rightarrow+\infty$, then we certainly know $q$ from (1.20). But then we know $v=v(q)$ and $\phi=\phi(q)$ in (1.18) and (1.19) and hence we know the asymptotics of $u(x)$ as $x \rightarrow-\infty$ from (1.17). Conversely, if we know the asymptotics of $u(x)$ as $x \rightarrow-\infty$, we certainly know $v>0$ from (1.17) and hence we know $q^{2}$ from (1.18), $q^{2}=1-e^{-2 \pi v}$. But then again from (1.17), we know $\phi$, and hence $\operatorname{sgn}(q)$ from (1.19). Thus we know $q$, and hence the asymptotics of the solution $u(x)$ as $x \rightarrow+\infty$ from (1.20). Finally note the similarity of the multiplier

$$
e^{x z-\frac{1}{3} z^{3}}
$$

for the Airy equation with the multiplier

$$
e^{i \theta}=e^{i\left(x z+\frac{4}{3} z^{3}\right)}
$$

in the RHP for PII. Setting $z \rightarrow i z$ in (1.21)

$$
e^{x z-\frac{1}{3} z^{3}} \rightarrow e^{i\left(x z+\frac{1}{3} z^{3}\right)}
$$

which agrees with (1.22) up to appropriate scalings.

Also note from (1.15) that PII is parameterized by parameters lying on a 2-dim variety: this corresponds to the fact that PII is second order. 
The fortunate and remarkable fact is that the class of problems in physics, mathematics, and engineering expressible in terms of a RHP is very broad and growing. Here is one more, with more to come!

The RHP for the MKdV equation (1.9) is as follows (see e.g., [16]): Let $\Sigma=\mathbb{R}$, oriented from $-\infty$ to $+\infty$. For fixed $x, t \in \mathbb{R}$ let

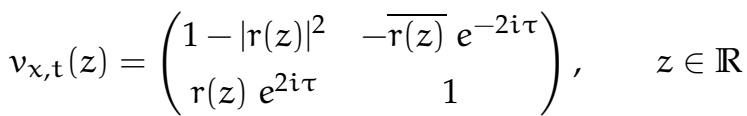

where $\tau=\tau_{x, t}(z)=x z+4 t z^{3}$ and $r=r(z)$ is a given function in $L^{\infty}(\mathbb{R}) \cap L^{2}(\mathbb{R})$ with

$$
\|r\|_{\infty}<1
$$

and

$$
\mathrm{r}(z)=-\overline{\mathrm{r}(-z)}, \quad z \in \mathbb{R} .
$$

There is a bijection from the initial data $u(x, t=0)=u_{0}(x)$ for MKdV onto such functions $r(z)$ - see later. The function $r(z)$ is called the reflection coefficient for $\mathfrak{u}_{0}$, see (4.13).

Let $m=m_{x, t}(z)$ be the solution of the normalized RHP $\left(\Sigma, v_{x, t}\right)$. Then

$$
u(x, t)=2\left(m_{1}(x, t)\right)_{12},
$$

is the solution of MKdV with initial condition $u(x, t=0)=u_{0}(x)$ corresponding to $r(z)$. Here

$$
\mathrm{m}_{\mathrm{x}, \mathrm{t}}(z)=\mathrm{I}+\frac{\mathrm{m}_{1}(x, \mathrm{t})}{z}+\mathrm{O}\left(\frac{1}{z^{2}}\right)
$$

as $z \rightarrow \infty$.

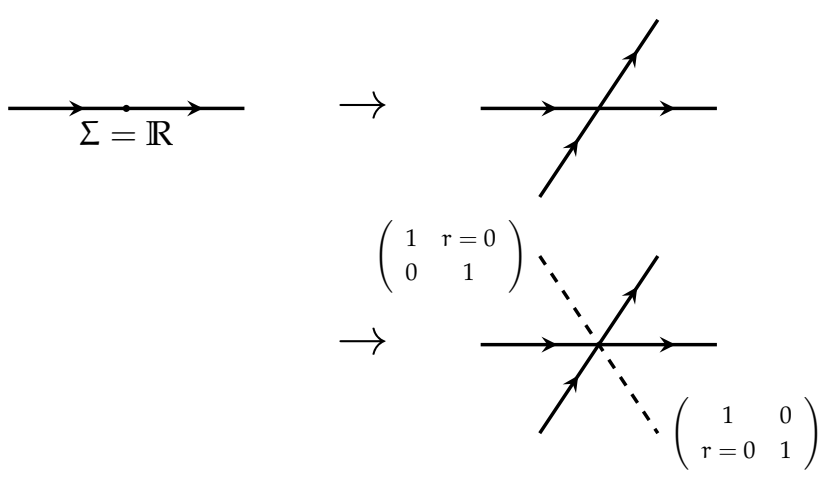

FIGURE 1.25. Obtaining six rays.

The asymptotic result (1.10) is obtained by applying the nonlinear steepest descent method to the $\operatorname{RHP}\left(\Sigma, v_{x, t}\right)$ in the region $|x| \leqslant c t^{1 / 3}$. In this case PII emerges as the RHP $\left(\Sigma, v_{x, t}\right)$ is "deformed" into the RHP $\left(\Sigma, v_{x}\right)$ in Figure 1.16.

As we will see, RHPs are useful not only for asymptotics, but also they can be used to determine symmetries and formulae/identities/equations, and also for analytical purposes. 


\section{Lecture 2}

We now consider some of technical issues that arise for RHPs, which are listed with bullet points above.

A key role in $\mathrm{RH}$ theory is played by the Cauchy operator. We first consider the case when $\Sigma=\mathbb{R}$. Here the Cauchy operator $C=C_{\mathbb{R}}$ is given by

$$
\mathrm{C} f(z)=\int_{\mathbb{R}} \frac{f(s)}{s-z} \text { đs, } \quad z \in \mathbb{C} / \mathbb{R}, \quad \text { đs }=\frac{d s}{2 \pi i}
$$

for suitable functions $f$ on $\mathbb{R}$ (General refs for the case $\Sigma=\mathbb{R}$, and also when $\Sigma=\{|z|=1\}$, are [19] and [23].) Assume first that $f \in \mathcal{S}(\mathbb{R})$, the Schwartz space of functions on $\mathbb{R}$. Let $z=x+i \epsilon, x \in \mathbb{R}, \epsilon>0$. Then

$$
\begin{aligned}
C f(x+i \epsilon) & =\int_{\mathbb{R}} \frac{f(s)}{s-x-i \epsilon} \text { đs } \\
& =\int_{\mathbb{R}} f(s) \frac{i \epsilon}{(s-x)^{2}+\epsilon^{2}} đ s+\int_{\mathbb{R}} f(s) \frac{s-x}{(s-x)^{2}+\epsilon^{2}} \text { đs } \\
& =\frac{1}{2} \int_{\mathbb{R}} f(s) \frac{1}{\pi} \frac{\epsilon}{(s-x)^{2}+\epsilon^{2}} d s+\frac{1}{2 \pi i} \int_{\mathbb{R}} f(s) \frac{s-x}{(s-x)^{2}+\epsilon^{2}} d s \\
& :=I+I I .
\end{aligned}
$$

Now

$$
\mathrm{I}=\mathrm{I}_{\epsilon}=\frac{1}{2} \int_{\mathbb{R}} \frac{f(x+\epsilon u)}{\pi\left(u^{2}+1\right)} d u .
$$

Then, by dominated convergence,

Write

$$
\lim _{\epsilon \downarrow 0} I_{\epsilon}=f(x) \frac{1}{2 \pi} \int_{\mathbb{R}} \frac{d u}{u^{2}+1}=\frac{1}{2} f(x) .
$$

$$
\mathrm{II}=\mathrm{II}_{\epsilon}=\mathrm{II}_{<\epsilon}+\mathrm{II}_{>\epsilon}
$$

where

$$
\mathrm{II}_{<\epsilon}=\frac{1}{2 \pi i} \int_{|\chi-s|<\epsilon} f(s) \frac{s-x}{(s-x)^{2}+\epsilon^{2}} d s
$$

and

$$
\mathrm{II}_{>\epsilon}=\frac{1}{2 \pi \mathrm{i}} \int_{|x-s|>\epsilon} f(s) \frac{s-x}{(s-x)^{2}+\epsilon^{2}} d s .
$$

As

$$
\frac{s-x}{(s-x)^{2}+\epsilon^{2}}
$$

is an odd function about $s=x, \mathrm{II}_{<\epsilon}$ can be written as

$$
\mathrm{II}_{<\epsilon}=\frac{1}{2 \pi \mathrm{i}} \int_{|s-x|<\epsilon} \frac{s-x}{(s-x)^{2}+\epsilon^{2}}(f(s)-f(x)) d s
$$

and so

$$
\begin{aligned}
\left|I_{<\epsilon}\right| & \leqslant \frac{1}{2 \pi}\left\|f^{\prime}\right\|_{L^{\infty}} \int_{|s-x|<\epsilon} \frac{(s-x)^{2}}{(s-x)^{2}+\epsilon^{2}} d s \\
& \leqslant\left\|f^{\prime}\right\|_{L^{\infty}} \frac{2 \epsilon}{2 \pi}
\end{aligned}
$$


which goes to 0 as $€ \downarrow 0$. Finally

$$
\begin{aligned}
\mathrm{II}_{>\epsilon} & =\frac{1}{2 \pi \mathrm{i}} \int_{|s-x|>\epsilon}\left(\frac{s-\chi}{(s-\chi)^{2}+\epsilon^{2}}-\frac{1}{s-\chi}\right) f(s) d s \\
& +\frac{1}{2 \pi \mathrm{i}} \int_{|s-x|>\epsilon} \frac{1}{s-\chi} f(s) d s \\
& =\mathrm{III}_{\epsilon}+\mathrm{IV}_{\epsilon}
\end{aligned}
$$

We have

$$
\begin{aligned}
\left|\mathrm{III}_{\epsilon}\right| & =\frac{1}{2 \pi}\left|\int_{|s-x|>\epsilon} \frac{\epsilon^{2}}{(s-x)^{2}+\epsilon^{2}} \frac{f(s)}{s-x} d s\right| \\
& =\frac{1}{2 \pi}\left|\int_{|u|>1} \frac{1}{u^{2}+1} \frac{f(x+\epsilon u)}{u} d u\right|
\end{aligned}
$$

and so as $\epsilon \downarrow 0$, again by dominated convergence,

$$
\left|\mathrm{III}_{\epsilon}\right| \rightarrow \frac{1}{2 \pi}|\mathrm{f}(\mathrm{x})|\left|\int_{|\mathfrak{u}|>1} \frac{\mathrm{du}}{\mathrm{u}\left(\mathrm{u}^{2}+1\right)}\right|=0
$$

as the final integrand is odd.

Thus we see that for $\Sigma=\mathbb{R}$ and $f \in \mathcal{S}(\mathbb{R})$

$$
C^{+} f(x) \equiv \lim _{\epsilon \downarrow 0} C f(x+i \epsilon)=\frac{1}{2} f(x)+\frac{i}{2} H f(x)
$$

where

$$
\operatorname{Hf}(x)=\lim _{\epsilon \downarrow 0} \frac{1}{\pi} \int_{|s-x|>\epsilon} \frac{f(s)}{x-s} d s .
$$

$\operatorname{Hf}(x)$ is called the Hilbert transform of $f$. Note that

$$
\begin{aligned}
\frac{1}{\pi} \int_{|s-x|>\epsilon} \frac{f(s)}{x-s} d s & =\frac{1}{\pi} \int_{|s-x|>1} \frac{f(s)}{x-s} d s \\
& +\frac{1}{\pi} \int_{\epsilon<|s-x|<1} \frac{f(s)-f(x)}{x-s} d s
\end{aligned}
$$

which converges to

$$
\frac{1}{\pi} \int_{|s-x|>1} \frac{f(s)}{x-s} d s+\frac{1}{\pi} \int_{|s-x|<1} \frac{f(s)-f(x)}{x-s} d s
$$

as $\epsilon \downarrow 0$, so that $\lim _{\epsilon \downarrow 0} \frac{1}{\pi} \int_{|s-x|>\epsilon} \frac{f(s)}{x-s}$ ds indeed exists pointwise for $f \in \mathcal{S}$.

Similarly one finds

$$
C^{-} f(x) \equiv \lim _{\epsilon \downarrow 0} C f(x-i \epsilon)=-\frac{1}{2} f(x)+\frac{i}{2} H f(x), \quad x \in \mathbb{R}
$$

and we obtain the fundamental relations for $f \in \mathcal{S}$

$$
C^{+} f-C^{-} f=f
$$

and

$$
\mathrm{C}^{+} \mathrm{f}+\mathrm{C}^{-} \mathrm{f}=\mathrm{iHf}
$$


Exercise 2.2. Show that the limits $C^{ \pm} f(x)=\lim _{\epsilon \downarrow 0} C f(x \pm i \epsilon)$ are in fact nontangential limits i.e. $\mathrm{C}^{+} \mathrm{f}(\mathrm{x})=\lim _{z^{\prime} \rightarrow \mathrm{x}} \mathrm{Cf}\left(z^{\prime}\right)$ where $z^{\prime}$ lies in a cone of arbitrary opening angle $\alpha<\pi$ (see Figure 2.3), and similarly for $\mathrm{C}^{-} f(x)$ (see refs. $[5,6,8]$ ).

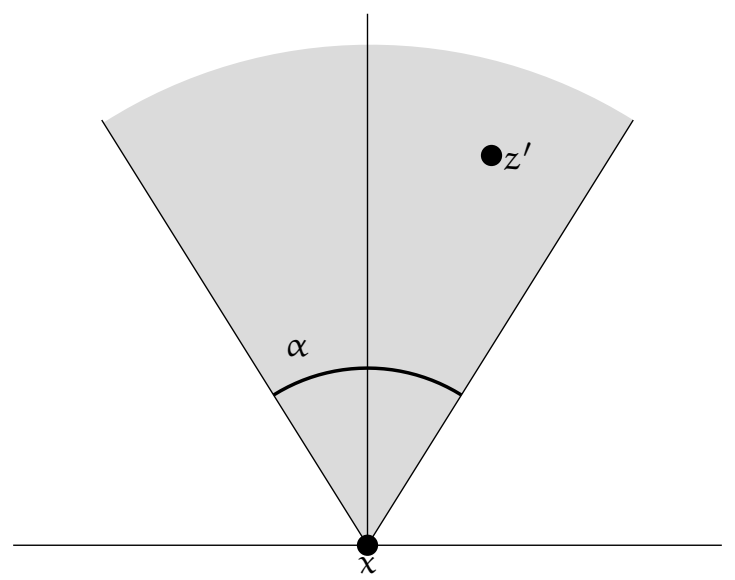

Figure 2.3. A cone of opening angle $\alpha$.

A critical property of the singular integral operator $\mathrm{H}$, and hence the operators $\mathrm{C}^{ \pm}$, is that, as we now show, $\mathrm{H}$ is a bounded operator from $\mathrm{L}^{\mathfrak{p}}(\mathbb{R}) \rightarrow \mathrm{L}^{\mathfrak{p}}(\mathbb{R})$ for all $1<p<\infty$. To prove the result for $\mathrm{L}^{2}$, recall that the Fourier transform

$$
\hat{f}(z)=\mathcal{F} f(z) \equiv \lim _{R \rightarrow \infty} \int_{-R}^{R} e^{-i z t} f(t) \frac{d t}{\sqrt{2 \pi}}, \quad z \in \mathbb{R}
$$

and the inverse Fourier transform

$$
\check{f}(x)=\mathcal{F}^{-1} f(x) \equiv \lim _{R \rightarrow \infty} \int_{-R}^{R} e^{i z t} f(t) \frac{d t}{\sqrt{2 \pi}}, \quad x \in \mathbb{R}
$$

are unitary maps

$$
\|\mathbf{f}\|_{L^{2}}=\|\hat{f}\|_{L^{2}}=\|\check{f}\|_{L^{2}}
$$

from $L^{2}$ onto $L^{2}$. Moreover

$\mathcal{F}$ and $\mathcal{F}^{-1}$ are indeed inverse to each other,

$$
(\hat{f})^{\vee}=f=(\check{f})^{\wedge}, \quad f \in L^{2}(\mathbb{R}) .
$$

For $f \in \mathcal{S}(\mathbb{R})$, fix $\in>0$, and set

$$
\left(C_{\epsilon} f\right)(x) \equiv C f(x+i \epsilon) .
$$

Then

$$
\begin{aligned}
\left(\mathcal{F} C_{\epsilon} f\right)(z) & =\lim _{R \rightarrow \infty} \int_{-R}^{R}\left(\int_{\mathbb{R}} \frac{f(s)}{s-x-i \epsilon} d s\right) e^{-i x z} \frac{d x}{\sqrt{2 \pi}} \\
& =\lim _{R \rightarrow \infty} \int_{\mathbb{R}} \frac{f(s)}{\sqrt{2 \pi}}\left(\int_{-R}^{R} \frac{e^{-i x z}}{s-x-i \epsilon} d x\right) d s,
\end{aligned}
$$

by Fubini's Theorem. 
Now for s fixed and R large, and $z>0$

$$
\begin{aligned}
& \int_{-R}^{R} \frac{e^{-i x z}}{s-x-i \epsilon} d x=-\int_{-R}^{R} \frac{e^{-i x z}}{x-(s-i \epsilon)} d x \\
& =\int_{\underbrace{}_{s-i \epsilon}}^{R} \frac{e^{-i x z}}{x-(s-i \epsilon)} d x-\int_{-R}^{R} \frac{e^{-i x z}}{x-(s-i \epsilon)} d x \\
& =e^{-i(s-i \epsilon) z}-\int_{-R}^{R} \frac{e^{-i x z}}{x-(s-i \epsilon)} d x .
\end{aligned}
$$

Exercise 2.5. Show that, for $z>0$, we have

$$
\lim _{R \rightarrow \infty} \int_{-R)^{R}} \frac{e^{-i x z}}{x-(s-i \epsilon)} d x=0 .
$$

Hence for $\mathrm{s}$ fixed and $z>0$

But we also have

$$
\lim _{R \rightarrow \infty} \int_{-R}^{R} \frac{e^{-i x z}}{s-x-i \epsilon} d x=e^{-i s z} e^{-\epsilon z} .
$$

Exercise 2.6. For $z>0$,

is bounded in s uniformly for $\mathrm{R}>0$.

$$
\int_{-R}^{R} \frac{e^{-i x z}}{s-x-i \epsilon} d x
$$

It follows that we may take the limit $R \rightarrow \infty$ in (2.4) in the s-integral and so for $z>0$

Exercise 2.7. Show, by a similar argument, that

$$
\mathcal{F} C_{\epsilon} f(z)=\int_{\mathbb{R}} f(s) e^{-i s z} e^{-\epsilon z} \frac{d s}{\sqrt{2 \pi}}=e^{-\epsilon z} \mathcal{F} f(z) .
$$

$$
\mathcal{F} C_{\epsilon} f(z)=0 \text { for } z<0
$$

Thus

$$
C_{\epsilon} f=\mathcal{F}^{-1}\left(\chi_{>0}(\cdot) e^{-\epsilon \cdot}\right) \mathcal{F f}
$$

where

$$
\chi>0(z) e^{-\epsilon z}= \begin{cases}e^{-\epsilon z} & \text { for } z>0 \\ 0 & \text { for } z<0\end{cases}
$$

Now as $\mathcal{S}(\mathbb{R})$ is dense in $L^{2}$, and as $\mathcal{F}^{-1}\left(\chi_{>0}(\cdot) e^{-\epsilon \cdot}\right) \mathcal{F}$ is clearly bounded in $L^{2}$ it follows that

$$
\mathrm{C}_{\epsilon} f \text { extends to a bounded operator on } \mathrm{L}^{2} \text {. }
$$

Moreover

$$
\hat{C} f(x) \equiv \mathcal{F}^{-1} \chi_{>0}(\cdot) \mathcal{F} f
$$


is clearly also a bounded operator in $\mathrm{L}^{2}$ and for $f \in \mathrm{L}^{2}$

$$
\begin{aligned}
\left\|\mathrm{C}_{\epsilon} \mathrm{f}-\hat{\mathrm{C}} \mathrm{f}\right\|_{\mathrm{L}^{2}} & =\left\|\mathcal{F}^{-1} \chi_{>0}(\cdot)\left(e^{-\epsilon \cdot}-1\right) \mathcal{F} \mathrm{f}\right\|_{\mathrm{L}^{2}} \\
& =\left\|\chi_{>0}(\cdot)\left(e^{-\epsilon \cdot}-1\right) \mathcal{F} \mathrm{f}\right\|_{\mathrm{L}^{2}}
\end{aligned}
$$

which converges to 0 as $€ \downarrow 0$, again by dominated convergence. In other words, for $f \in \mathrm{L}^{2}$,

$$
C f(x+i \epsilon)=\int_{\mathbb{R}} \frac{f(s)}{s-x-i \epsilon} đ s \rightarrow \hat{C} f(x) \text { in } L^{2}(d x) .
$$

In particular, it follows by general measure theory, that for some sequence $\epsilon_{\mathrm{n}} \downarrow 0$

$$
\mathrm{C} f\left(x+i \epsilon_{n}\right) \rightarrow \hat{C} f(x)
$$

pointwise a.e. In particular (2.8) holds for $f \in \mathcal{S}(\mathbb{R})$. But then by our previous calculations, $C f\left(x+i \epsilon_{n}\right)$ converges pointwise for all $x$, and we conclude that for $f \in \mathcal{S}$ and a.e. $x$

$$
C^{+} f(x)=\frac{1}{2} f(x)+\frac{i}{2} H f(x)=\hat{C} f(x) \quad \text { a.e. } x .
$$

Thus $\mathrm{C}^{+} \mathrm{f}$ and, hence $\mathrm{Hf}$, extend to bounded operators on $\mathrm{L}^{2}(\mathbb{R})$ and

and so

$$
\frac{1}{2} \hat{f}+\frac{i}{2} \mathcal{F} H f=\mathcal{F} \hat{C} f=x_{>0} \hat{f}
$$

$$
\begin{aligned}
\mathcal{F H} f(z) & =\frac{2}{i}\left(x_{>0}(z)-\frac{1}{2}\right) \hat{f}(z) \\
& =-i \operatorname{sgn}(z) \hat{f}(z)
\end{aligned}
$$

where $\operatorname{sgn}(z)=+1$ if $z>0$ and $\operatorname{sgn}(z)=-1$ if $z<0$.

We have shown the following: For $f \in \mathrm{L}^{2}$,

$$
C^{+} f=\frac{f}{2}+\frac{i}{2} H f=\frac{f}{2}+\frac{1}{2}(\hat{f} \operatorname{sgn}(\cdot))^{\vee}
$$

and similarly

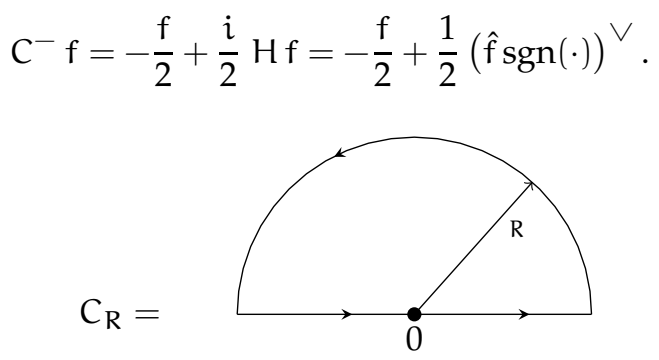

FIGURE 2.9. A semi-circle in the upper-half plane.

The following argument of Riesz shows that in fact $C^{ \pm}$, and hence $H$, are bounded in $L^{p}(\mathbb{R})$, for all $1<p<\infty$. Consider first the case $p=4$. Suppose $\mathrm{f} \in \mathrm{C}_{0}^{\infty}(\mathbb{R})$, the infinitely differentiable functions with compact support. Then as $z \rightarrow \infty$,

$$
\mathrm{C} f(z)=\int_{\mathbb{R}} \frac{\mathrm{f}(\mathrm{s})}{\mathrm{s}-\mathrm{z}} \mathrm{đs}=\mathrm{O}\left(\frac{1}{z}\right)
$$


and $\mathrm{C} f(z)$ is continuous down to the axis. By Cauchy's theorem

$$
\int_{C_{R}}(C f(z))^{4} d z=0
$$

where $C_{R}$ is given in Figure 2.9, and as

$$
\int_{R}(\mathrm{C} f(z))^{4} d z \rightarrow 0 \text { as } R \rightarrow \infty,
$$

we conclude that

$$
\int_{\mathbb{R}}\left(C^{+} f(x)\right)^{4} d x=0 .
$$

But then as $\mathrm{C}^{+} \mathrm{f}=\frac{\mathrm{f}}{2}+\frac{i}{2} \mathrm{H} f$ we obtain

$$
0=\int_{\mathbb{R}}\left(f^{4}+4 f^{3}(H f) i+6 f^{2}(H f)^{2} i^{2}+4 f(H f)^{3} i^{3}+(H f)^{4} i^{4}\right) d x .
$$

Now suppose that $\mathrm{f}$ is real. Then $\mathrm{Hf}$ is real and the real part of (2.10) yields

$$
0=\int_{\mathbb{R}}\left(f^{4}-6 f^{2}(H f)^{2}+(H f)^{4}\right) d x,
$$

hence

$$
\begin{aligned}
\int_{\mathbb{R}}(H f)^{4} d x & =6 \int_{\mathbb{R}} f^{2}(H f)^{2} d x-\int_{\mathbb{R}} f^{4} d x \\
& \leqslant 6\left(\int \frac{c}{2} f^{4} d x+\int_{\mathbb{R}} \frac{1}{2 c}(H f)^{4} d x\right)-\int_{\mathbb{R}} f^{4} d x
\end{aligned}
$$

for any $c>0$. Take $c=6$. Then

$$
\frac{1}{2} \int_{\mathbb{R}}(H f)^{4} \mathrm{~d} x \leqslant(18-1) \int_{\mathbb{R}} f^{4} \mathrm{~d} x
$$

or

$$
\int_{\mathbb{R}}(H f)^{4} d x \leqslant 34 \int_{\mathbb{R}} f^{4} d x
$$

The case when $f$ is complex valued is handled by taking real and imaginary parts. Thus, by density, $\mathrm{H}$ maps $\mathrm{L}^{4}$ boundedly to $\mathrm{L}^{4}$.

Exercise 2.11. Show that $\mathrm{H}$ maps $\mathrm{L}^{p} \rightarrow \mathrm{L}^{p}$ for all $1<p<\infty$. Hints:

(1) Show that the above argument works for all even integers $p$.

(2) Show that the result follows for all $p \geqslant 2$ by interpolation.

(3) Show that the result for $1<p<2$ now follows by duality.

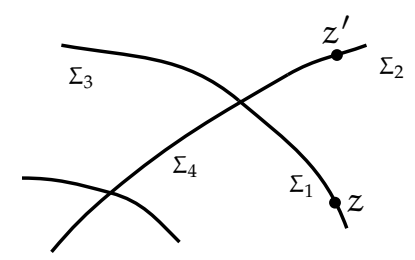

FigURE 2.12. Contours that self-intersect. 
Exercise 2.13. Show that $H$ is not bounded from $L^{1} \rightarrow L^{1}$. (However $H$ maps $\mathrm{L}^{1} \rightarrow$ weak $\mathrm{L}^{1}$.) As indicated in Lecture 1 , RHPs take place on contours which self-intersect (see Figure 2.12).

We will need to know, for example, that if $\mathrm{f}$ is supported on $\Sigma_{1}$, say, and we consider

$$
\mathrm{Cf}\left(z^{\prime}\right)=\int_{\Sigma_{1}} \frac{f(z)}{z-z^{\prime}} \mathrm{d} z
$$

for $z^{\prime} \in \Sigma_{2}$, say, then $\operatorname{Cf}\left(z^{\prime}\right) \in \mathrm{L}^{2}\left(\Sigma_{2}\right)$ if $\mathrm{f} \in \mathrm{L}^{2}\left(\Sigma_{1}\right)$. Here is a prototype result which one can prove using the Mellin transform, which we recall is the Fourier transform for the multiplicative group $\{x>0\}$. We have [5, p. 88] the following:

For $f \in \mathrm{L}^{2}(0, \infty)$ and $r>0$, set

$$
C_{\theta} f(r)=\int_{0}^{\infty} \frac{f(s)}{s-\hat{z} r} \text { đs, } \quad \hat{z}=e^{i \theta}
$$

where $0<\theta<2 \pi$. Then

$$
\left\|C_{\theta} f\right\|_{L^{2}(d r)} \leqslant c_{\theta}\|f\|_{L^{2}(d s)}
$$

where

$$
\mathrm{c}_{\theta}=\gamma^{\gamma}(1-\gamma)^{1-\gamma}, \quad \gamma=\frac{\theta}{2 \pi} .
$$

One can also show that for any $1<p<\infty$

$$
\left\|C_{\theta} f\right\|_{L^{p}(d r)} \leqslant C_{\theta, p}\|f\|_{L^{p}(d s)}
$$

for some $c_{\theta, p}<\infty$.

Results such as (2.14) are useful in many ways. For example, we have the following result.

Theorem 2.15. Suppose $f \in H^{1}(\mathbb{R})=\left\{f \in L^{2}(\mathbb{R}): f^{\prime} \in L^{2}(\mathbb{R})\right\}$. Then $C f(z)$ is uniformly Hölder $-\frac{1}{2}$ in $\overline{\mathbf{C}}_{+}$and in $\overline{\mathbf{C}}_{-}$. In particular, $\mathrm{Cf}$ is continuous down to the axis in $\mathbb{C}_{+}$and in $\mathbb{C}_{-}$.

Proof. For $z \in \mathbb{C} \backslash \mathbb{R}$

$$
\begin{aligned}
\frac{d}{d z} \operatorname{Cf}(z) & =\int_{\mathbb{R}} \frac{f(s)}{(s-z)^{2}} \text { đs }=-\int_{\mathbb{R}}\left(\frac{d}{d s}\left(\frac{1}{s-z}\right)\right) f(s) \text { đs } \\
& =\int_{\mathbb{R}} \frac{f^{\prime}(s)}{s-z} \text { đs. }
\end{aligned}
$$

Now suppose $z^{\prime}, z^{\prime \prime} \in \mathbb{C}_{+}$, and the straight line $L$ through $z^{\prime}, z^{\prime \prime}$ intersects the line $\mathbb{R}$ at $x$ at an angle $\theta$ as in Figure 2.16.

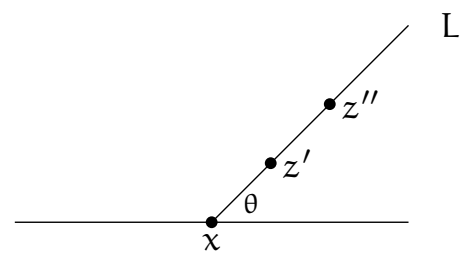

FIGURE 2.16. A line intersecting $\mathbb{R}$. 
Then as $\int_{\mathbb{R}} \frac{f^{\prime}(s)}{s-z}$ đs $=\int_{x}^{\infty} \frac{f^{\prime}(s)}{s-z}$ đs $+\int_{-\infty}^{x} \frac{f^{\prime}(s)}{s-z}$ đs, and as $f^{\prime} \in L^{2}(-\infty, x) \oplus$ $\mathrm{L}^{2}(x, \infty)$ it follows from (2.14) that

$$
\int_{0}^{\infty}\left|\frac{\mathrm{d}}{\mathrm{d} z} \mathrm{Cf}\left(\mathrm{r} e^{i \theta}\right)\right|^{2} \mathrm{dr}=\int_{0}^{\infty}\left|C \mathrm{f}^{\prime}\left(r e^{i \theta}\right)\right|^{2} \mathrm{dr} \leqslant \mathrm{c}\left\|\mathrm{f}^{\prime}\right\|_{\mathrm{L}^{2}}
$$

But

$$
\begin{aligned}
& \left|\operatorname{Cf}\left(z^{\prime \prime}\right)-\operatorname{Cf}\left(z^{\prime}\right)\right|=\left|\int_{z^{\prime} \rightarrow z^{\prime \prime} \text { in } L} \frac{d}{d z} \operatorname{Cf}(z) d z\right| \\
& \quad \leqslant\left|z^{\prime \prime}-z^{\prime}\right|^{\frac{1}{2}}\left\|\frac{d}{d z} C f\right\|_{L^{2}(0, \infty)} \leqslant c\left|z^{\prime \prime}-z^{\prime}\right|^{\frac{1}{2}}\left\|f^{\prime}\right\|_{L^{2}(\mathbb{R})} .
\end{aligned}
$$

We now consider general contours $\Sigma \subset \overline{\mathbb{C}}=\mathbb{C} \cup\{\infty\}$, which are composed curves: By definition a composed curve $\Sigma$ is a finite union of $\operatorname{arcs}\left\{\Sigma_{i}\right\}_{i=1}^{n}$ which can intersect only at their end points. Each $\operatorname{arc} \Sigma_{i}$ is homeomorphic to an interval $\left[a_{i}, b_{i}\right] \subset \mathbb{R}:$

$$
\left\{\begin{aligned}
\varphi_{i}:\left[a_{i}, b_{i}\right] & \rightarrow \Sigma_{i} \subset \overline{\mathbb{C}} \\
{\left[a_{i}, b_{i}\right] \ni t } & \rightarrow \varphi_{i}(t) \in \Sigma_{i}, \varphi_{i}\left(a_{i}\right) \neq \varphi_{i}\left(b_{i}\right)
\end{aligned}\right.
$$

Here $\overline{\mathbb{C}}$ has the natural topology generated by the sets $\left\{|z|<R_{1}\right\}, \quad\left\{|z|>R_{2}\right\}$ where $R_{1}, R_{2}>0$. A loop, in particular the unit circle $T=\{|z|=1\}$, is a composed curve on the understanding that it is a union of (at least) two arcs.

Although it is possible, and sometimes useful, to consider other function spaces (e.g. Hölder continuous functions), we will only consider RHPs in the sense of $\mathrm{L}^{\mathrm{p}}(\Sigma)$ for $1<\mathrm{p}<\infty$.

So the first question is "What is $\mathrm{L}^{\mathrm{p}}(\Sigma)$ ?". The natural measure theory for each $\operatorname{arc} \Sigma_{i}$ is generated by arc length measure $\mu$ as follows. If $z_{0}=\varphi\left(t_{0}\right)$ and $z_{\mathrm{n}}=\varphi\left(\mathrm{t}_{\mathrm{n}}\right)$ are the end-points of some arc $\Sigma \subset \mathbb{C}$, and $z_{0}, z_{1}, \ldots, z_{\mathrm{n}}$ is any partition of $\left[z_{0}, z_{n}\right]=\left\{\varphi(t): t_{0} \leqslant t \leqslant t_{n}\right\}$ (we assume $z_{i+1}$ succeeds $z_{i}$ in the ordering induced on $\Sigma$ by $\varphi$, symbolically $z_{i}<z_{i+1}$, etc.) then

$$
\mathrm{L}=\mathrm{L}_{\left[z_{0}, z_{n}\right]} \equiv \sup _{\text {all partitions }\left\{z_{i}\right\}} \sum_{i=0}^{n-1}\left|z_{i+1}-z_{i}\right| .
$$

If $\mathrm{L}<\infty$ we say that the arc $\Sigma=\left[z_{0}, z_{n}\right]$ is rectifiable and $\mathrm{L}_{\left[z_{0}, z_{n}\right]}$ is its arc length. We will only consider composed curves $\Sigma$ that are locally rectifiable i.e. for any $\mathrm{R}>0, \Sigma \cap\{|z|<\mathrm{R}\}$ is rectifiable (note that the latter set is an at most countable union of simple arcs and rectifiability of the set means that the sum of the arc lengths of these arcs is finite. In particular, the unit circle $\mathbb{T}$ as a union of 2 rectifiable subarcs, is rectifiable, and $\mathbb{R}$ is locally rectifiable.) For any interval $[\alpha, \beta)$ on $\Sigma_{i} \subset \mathbb{C}$ (the case where $\Sigma_{i}$ passes through $\infty$, must be treated separately - exercise!) define

$$
\mu_{i}([\alpha, \beta))=\text { arc length } \alpha \rightarrow \beta \text {. }
$$

Now the sets $\left\{[\alpha, \beta): \alpha<\beta\right.$ on $\left.\Sigma_{i}\right\}$ form a semi-algebra (see [30]) and hence $\mu_{i}$ can be extended to a complete measure on a $\sigma$-algebra $\mathcal{A}$ containing the Borel sets on $\Sigma_{i}$. The restriction of the measure to the Borel sets is unique. For $1 \leqslant p<\infty$, 
we can define $L^{p}\left(\Sigma_{i}, d \mu_{i}\right)$ to be the set of $f$ measurable with respect to $\mathcal{A}$ on $\Sigma$ for which,

$$
\int_{\Sigma_{i}}|f(z)|^{p} \mathrm{~d} \mu_{i}(z)<\infty,
$$

and then all the "usual" properties go through. One usually writes $\mathrm{d} \mu=|\mathrm{d} z|$. For $\Sigma=\bigcup_{i=1}^{n} \Sigma_{i}, L^{p}(\Sigma, d \mu)$ is simply the direct sum of $L^{p}\left(\Sigma_{i}, d \mu_{i}\right)_{i=1}^{n}$.

Exercise 2.17. $|\mathrm{d} z|$ is also equal to Hausdorff-1 measure on $\Sigma_{1}$.

Note that if $\Sigma_{1}=\mathbb{R}$ and $\Sigma_{2}=\left\{\left(x, x^{3} \sin \frac{1}{x}\right): x \in \mathbb{R}\right\}$ then $\Sigma=\Sigma_{1} \cup \Sigma_{2}$ is not a composed curve, although $\Sigma_{1}$ and $\Sigma_{2}$ are both locally rectifiable.

For $\Sigma$ as above we define the Cauchy operator for $h \in \mathrm{L}^{\mathrm{p}}(\Sigma,|\mathrm{d} z|), 1 \leqslant p<\infty$, by

$$
\mathrm{Cf}(z)=\mathrm{C}_{\Sigma} \mathrm{f}(z)=\int_{\Sigma} \frac{\mathrm{f}(\zeta)}{\zeta-z} đ \zeta, \quad z \in \mathbb{C} \backslash \Sigma .
$$

Given the homeomorphisms $\varphi_{i}:\left[a_{i}, b_{i}\right] \rightarrow \Sigma_{i}$, the contour $\Sigma$ carries a natural orientation, and the integral here is a line integral following the orientation; if we parametrize the $\operatorname{arcs} \Sigma_{i}$ in $\Sigma$ by arc length $s$,

$$
0 \leqslant s \leqslant s_{i}, \quad \zeta=\zeta(s), \quad \text { then } \quad\left|\frac{d \zeta(s)}{d s}(s)\right|=1 \quad \text { (why?) }
$$

and (2.18) is a sum over its subarcs $\Sigma_{i}$ of integrals of the form

$$
\int_{0}^{s_{i}} \frac{f(\zeta(s))}{\zeta(s)-z} \frac{d \zeta(s)}{d s} \text { đs, } \quad z \in \mathbb{C} \backslash \Sigma
$$

for each $i$, the integrand (clearly) lies in $L^{p}\left(d s:\left[0, s_{i}\right)\right)$.

Now the fact of the matter is that many of the properties that were true for $C_{\Sigma}$ when $\Sigma=\mathbb{R}$, go through for $C_{\Sigma}$ in the general situation. (See, in particular, [24].) In particular for $f \in \mathrm{L}^{\mathrm{p}}(\Sigma, \mathrm{d} \mu)$, the non-tangential limits

$$
C_{\Sigma}^{ \pm} f(z)=\lim _{z^{\prime} \rightarrow z^{ \pm}} C_{\Sigma} f\left(z^{\prime}\right)
$$

exist pointwise a.e. on $\Sigma$. Figure 2.20 demonstrates non-tangential limits.

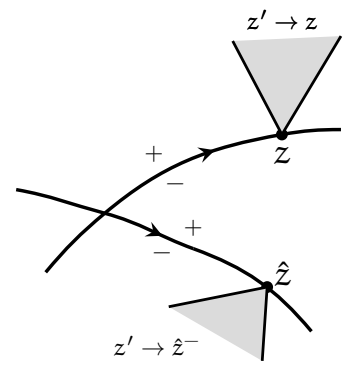

Figure 2.20. Non-tangential limits.

Note that as $\Sigma_{i}$ is locally rectifiable, the tangent vector to the arc $\frac{\mathrm{d} \zeta}{\mathrm{ds}}$ exists at a.e. point $\zeta=\zeta(\mathrm{s})$ : the normal to $\frac{\mathrm{d} \zeta}{\mathrm{ds}}$ bisects the cone. 


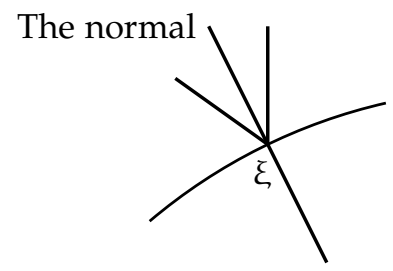

Figure 2.21. A contour and its normal.

\section{Moreover,}

$$
C_{\Sigma}^{ \pm} f(z)= \pm \frac{1}{2} f(z)+\frac{i}{2} H f(z)
$$

where the Hilbert transform is now given by

$$
\operatorname{Hf}(z)=\frac{1}{\pi} \lim _{\epsilon \downarrow 0} \int_{\substack{|s-z|>\epsilon \\ s \in \Sigma}} \frac{f(s)}{z-s} d s, \quad z \in \Sigma
$$

and the points $z \in \Sigma$ for which the non-tangential limits (2.19) exists are precisely the points for which the limit in (2.22) exists.

Again, for $f \in L^{p}(\Sigma, d \mu)$ with $1 \leqslant p<\infty$,

$$
C^{+} f(z)-C^{-} f(z)=f(z)
$$

and

$$
\mathrm{C}^{+} \mathrm{f}(z)+\mathrm{C}^{-} \mathrm{f}(z)=i \mathrm{Hh}(z) .
$$

The following issue is crucial for the analysis of RHPs:

Question. For which locally rectifiable contours $\Sigma$ are the operators $\mathrm{C}^{ \pm}$and $\mathrm{H}$ bounded in $\mathrm{L}^{\mathrm{p}}, 1<\mathrm{p}<\infty$ ?

Quite remarkably, it turns out that there are necessary and sufficient conditions on a simple rectifiable curve for $\mathrm{C}^{ \pm}, \mathrm{H}$ to be bounded in $\mathrm{L}^{\mathrm{p}}(\Sigma), 1<\mathrm{p}<\infty$. The result is due to many authors, starting with Calderón [7], and then Coifman, Meyer and McIntosh [9], with Guy David [10] (see [6] for details and historical references) making the final decisive contribution.

Let $\Sigma$ be a simple, rectifiable curve in $\mathbb{C}$. For any $z \in \Sigma$, and any $r>0$, let

$$
\ell_{\mathrm{r}}(z)=\text { arc length of }\left(\Sigma \cap \mathrm{D}_{\mathrm{r}}(z)\right)
$$

where $D_{r}(z)$ is the ball of the radius $r$ centered at $z$, see Figure 2.23.

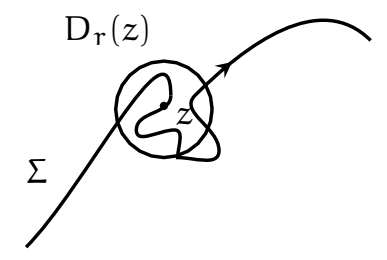

FIGURE 2.23. A ball $D_{\mathrm{r}}(z)$ of radius $r$ centered at $z$. 
Set

$$
\lambda=\lambda_{\Sigma}=\sup _{z \in \Sigma, \mathrm{r}>0} \frac{\ell_{\mathrm{r}}(z)}{\mathrm{r}} .
$$

Theorem 2.24. Suppose $\lambda_{\Sigma}<\infty$. Then for any $1<p<\infty$, the limit in (2.22) exists for a.e. $z \in \Sigma$ and defines a bounded operator for any $1<p<\infty$

$$
\|\mathrm{H} f\|_{L^{p}} \leqslant c_{p}\|f\|_{L^{p}}, \quad f \in L^{p}, \quad c_{p}<\infty .
$$

Conversely if the limit in (2.22) exists a.e. and defines a bounded operator $\mathrm{H}$ in $\mathrm{L}^{\mathrm{p}}(\Sigma)$ for some $1<\mathrm{p}<\infty$, then $\mathrm{H}$ gives rise to a bounded operator for all $\mathrm{p}, 1<\mathrm{p}<\infty$, and $\lambda_{\Sigma}<\infty$.

An excellent reference for the above Theorem, and more, is [6].

Remarks. Additional remarks:

(1) Locally rectifiable curves $\Sigma$ for which $\lambda=\lambda_{\Sigma}<\infty$ are called Carleson curves,

(2) the constant $c_{p}$ in (2.25) has the form $c_{p}=\phi_{p}\left(\lambda_{\Sigma}\right)$ for some continuous, increasing function, $\phi_{p}(t) \geqslant 0$, independent of $\Sigma$, such that $\phi_{p}(0)=0$.

The fact that $\phi_{p}$ is independent of $\Sigma$, is very important for the nonlinear steepest descent method, where one deforms curves in a similar way to the classical steepest descent method for integrals.

Carleson curves are sometimes called AD-regular curves: the A and D denote Ahlfors and David. To get some sense of the subtlety of the above result, consider the following curve $\Sigma$ with a cusp at the origin (see Figure 2.26):

$$
\Sigma=\{0 \leqslant x \leqslant 1, y=0\} \cup\left\{\left(x, x^{2}\right): 0 \leqslant x \leqslant 1\right\} .
$$

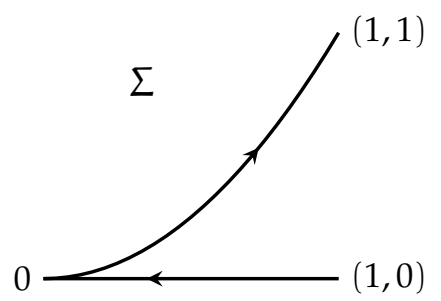

Figure 2.26. A cusp at the origin.

Clearly $\lambda_{\Sigma}<\infty$ so that the Hilbert transform $\mathrm{H}_{\Sigma}$ is bounded in $\mathrm{L}^{\mathrm{p}}, 1<\mathrm{p}<\infty$.

Exercise 2.27. For $\Sigma$ in Figure 2.26, prove directly that $\mathrm{H}_{\Sigma}$ is bounded in $\mathrm{L}^{2}$. The presence of the cusp makes the proof surprisingly difficult.

\section{Lecture 3}

We now make the notion of a RHP precise (see $[8,17,28])$. Let $\Sigma$ be a composite, oriented Carleson contour in $\mathbb{C}$ and let $v: \Sigma \rightarrow \mathrm{GL}(n, \mathbb{C})$ be a jump matrix on $\Sigma$, 
with $v, v^{-1} \in \mathrm{L}^{\infty}(\Sigma)$. Let $\mathrm{Ch}(z)=\mathrm{C}_{\Sigma} \mathrm{h}(z), \mathrm{C}_{\Sigma}^{ \pm} \mathrm{h}, \mathrm{H}_{\Sigma} \mathrm{h}$ be the associated Cauchy and Hilbert operators.

We say that a pair of $L^{p}(\Sigma)$ function $f_{ \pm} \in \partial C\left(L^{p}\right)$ if there exists a (unique) function $h \in L^{p}(\Sigma)$ such that

$$
\mathrm{f}_{ \pm}(z)=\left(\mathrm{C}^{ \pm} \mathrm{h}\right)(z), \quad z \in \Sigma .
$$

In turn we call $\mathrm{f}(z) \equiv \mathrm{Ch}(z), \quad z \in \mathbb{C} \backslash \Sigma$, the extension of $f_{ \pm}=C^{ \pm} h \in \partial C\left(L^{p}\right)$ off $\Sigma$.

Definition 3.1. Fix $1<p<\infty$. Given $\Sigma, v$ and a measurable function $f$ on $\Sigma$, we say that $\mathrm{m}_{ \pm} \in \mathrm{f}+\partial \mathrm{C}\left(\mathrm{L}^{\mathrm{p}}\right)$ solves an inhomogeneous RHP of the first kind (IRHP1 $1_{\mathrm{p}}$ ) if

$$
\mathrm{m}_{+}(z)=\mathrm{m}_{-}(z) v(z), \quad z \in \Sigma .
$$

Definition 3.2. Fix $1<p<\infty$. Given $\Sigma, v$ and a function $F \in L^{p}(\Sigma)$, we say that $\mathrm{M}_{ \pm} \in \partial \mathrm{C}\left(\mathrm{L}^{\mathrm{p}}\right)$ solves an inhomogeneous RHP of the second kind (IRHP2 $\left.2_{\mathrm{p}}\right)$ if

$$
M_{+}(z)=M_{-}(z) v(z)+F(z), \quad z \in \Sigma .
$$

Recall that $m$ solves the normalized $\operatorname{RHP}(\Sigma, v)$ if, at least formally,

- $m(z)$ is a $n \times n$ analytic function in $\mathbb{C} \backslash \Sigma$,

- $\mathrm{m}_{+}(z)=\mathrm{m}_{-}(z) v(z), \quad z \in \Sigma$,

- $\mathrm{m}(z) \rightarrow \mathrm{I}$ as $z \rightarrow \infty$.

More precisely, we make the following definition.

Definition 3.4. Fix $1<p<\infty$. We say that $\mathrm{m}_{ \pm}$solves the normalized $R H P(\Sigma, v)_{p}$ if $\mathrm{m}_{ \pm}$solves the IRHP1 $1_{\mathrm{p}}$ with $\mathrm{f} \equiv \mathrm{I}$.

In the above definition, if $\mathrm{m}_{ \pm}-\mathrm{I} \in \mathrm{C}^{ \pm} \mathrm{h}$, then clearly the extension

$$
\mathrm{m}(z)=\mathrm{I}+\mathrm{Ch}(z), \quad z \in \mathbb{C} \backslash \Sigma,
$$

off $\Sigma$ solves the normalized RHP in the formal sense of (3.3).

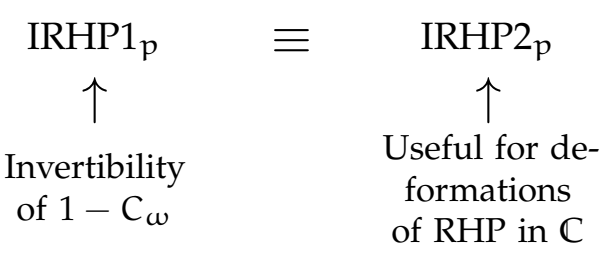

FIGURE 3.5. The uses of IRHP $1_{p}$ and IRHP $2_{p}$.

Let

$$
\begin{gathered}
v=\left(v^{-}\right)^{-1} v^{+}=\left(\mathrm{I}-\omega^{-}\right)^{-1}\left(\mathrm{I}+\omega^{+}\right) \\
\omega^{+} \equiv v^{+}-\mathrm{I}, \quad \omega^{-} \equiv \mathrm{I}-v^{-},
\end{gathered}
$$

be a pointwise a.e. factorization of $v$, i.e., $v(x)=\left(v^{-}(x)\right)^{-1} v^{+}(x)$ for a.e. $x$, with $v^{ \pm},\left(v^{ \pm}\right)^{-1} \in \mathrm{L}^{\infty}$, and let $\omega=\left(\omega^{-}, \omega^{+}\right)$. Let $\mathrm{C}_{\omega}$ denote the basic associated 
operator

$$
\mathrm{C}_{\omega} \mathrm{h} \equiv \mathrm{C}^{+}\left(\mathrm{h} \omega^{-}\right)+\mathrm{C}^{-}\left(\mathrm{h} \omega^{+}\right)
$$

acting on $L^{p}(\Sigma)-n \times n$ matrix valued functions $h$. As $\omega^{ \pm} \in L^{\infty}, C_{\omega} \in \mathcal{L}\left(L^{p}\right)$, the bounded operators on $L^{p}$, for all $1<p<\infty$. The utility of IRHP1 $p$ and IRHP $2_{p}$ will soon become clear, see Figure 3.5.

Theorem 3.6. If $\mathrm{f}$ and $v$ are such that $\mathrm{f}(v-\mathrm{I}) \in \mathrm{L}^{\mathrm{p}}(\Sigma)$ for some $<\mathrm{p}<\infty$, then

$$
m_{ \pm}=M_{ \pm}+f
$$

solves IRHP1 $1_{p}$ if $M_{ \pm}$solves IRPH $2_{p}$ with $F=f(v-I)$. Conversely if $F \in L^{p}(\Sigma)$, then

$$
M_{+}=m_{+}+F, \quad M_{-}=m_{-}
$$

solves IRHP2 $2_{p}$ if $\mathrm{m}_{ \pm}$solves IRHP $1_{\mathrm{p}}$ with $\mathrm{f}=\mathrm{C}^{-} \mathrm{F}$.

The first part of this result is straightforward: Suppose $M_{ \pm} \in \partial C\left(L^{p}\right)$ solves $M_{+}=M_{-} v+F$ on $\Sigma$ with $F=f(v-I) \in L^{p}$. Then

$$
M_{+}=M_{-} v+f(v-I)=\left(M_{-}+f\right) v-f
$$

or $m_{+}=m_{-} v$ with $m_{ \pm}=f+M_{ \pm} \in f+\partial C\left(L^{p}\right)$. The converse is more subtle and is left as an exercise:

Exercise 3.7. Show IRHP $1_{p} \Rightarrow I R H P 2_{p}$.

We now show that the RHPs IRHP $1_{p}$ and IRHP2 $2_{p}$, and, in particular, the normalized RHP $(\Sigma, v)_{p}$ are intimately connected with the singular integral operator $1-\mathrm{C}_{\omega}$.

Let $f \in L^{p}(\Sigma)$ and let $m_{ \pm}=f+C^{ \pm} h$ for some $h \in L^{p}(\Sigma)$. Also suppose $\mathrm{m}_{+}=\mathrm{m}_{-} v=\mathrm{m}_{-}\left(v^{-}\right)^{-1} v^{+}$. Set

$$
\mu=\mathrm{m}_{-}\left(v^{-}\right)^{-1}=\mathrm{m}_{+}\left(v^{+}\right)^{-1} \in \mathrm{L}^{\mathrm{p}}(\Sigma)
$$

and define

$$
\mathrm{H}(z)=\left(\mathrm{C}\left(\mu\left(\omega^{+}+\omega^{-}\right)\right)\right)(z), \quad z \in \mathbb{C} \backslash \Sigma .
$$

Then we have on $\Sigma$, using $\mathrm{C}^{+}-\mathrm{C}^{-}=1$

$$
\begin{aligned}
\mathrm{H}_{+} & =\mathrm{C}^{+}\left(\mu\left(\omega^{+}+\omega^{-}\right)\right)=\mathrm{C}^{+} \mu \omega^{+}+\mathrm{C}^{+} \mu \omega^{-} \\
& =\mathrm{C}^{+} \mu \omega^{-}+\mathrm{C}^{-} \mu \omega^{+}+\mu \omega^{+} \\
& =C_{\omega} \mu+\mu \omega^{+}=\left(C_{\omega}-1\right) \mu+\mu\left(\mathrm{I}+\omega^{+}\right) \\
& =\left(C_{\omega}-1\right) \mu+\mu v^{+}=\left(C_{\omega}-1\right) \mu+m_{+} .
\end{aligned}
$$

i.e. $H_{+}=\left(C_{\omega}-I\right) \mu+m_{+}$.

Similarly

$$
H_{-}=\left(C_{\omega}-1\right) \mu+m_{-} .
$$

Thus

$$
m_{ \pm}-f-H_{ \pm}=\left(1-C_{\omega}\right) \mu-f .
$$


But $m_{ \pm}-f-H_{ \pm} \in \partial C\left(L^{p}\right)$; i.e. $m_{ \pm}-f-H_{ \pm}=C^{ \pm} h$ for some $h \in L^{p}$. However, from (3.8)

$$
\mathrm{C}^{+} \mathrm{h}=\mathrm{C}^{-} \mathrm{h} \quad \Rightarrow \quad \mathrm{h}=\mathrm{C}^{+} \mathrm{h}-\mathrm{C}^{-} \mathrm{h}=0 .
$$

We conclude that $\left(1-C_{\omega}\right) \mu=f, \mu \in L^{p}$.

Conversely, if $\mu \in \mathrm{L}^{\mathrm{p}}(\Sigma)$ solves $\left(1-\mathrm{C}_{\omega}\right) \mu=\mathrm{f}$, then the above calculations show that $\mathrm{H} \equiv \mathrm{C}\left(\mu\left(\omega^{+}+\omega^{-}\right)\right)$satisfies

$$
\mathrm{H}_{ \pm}=-\mathrm{f}+\mu v^{ \pm} .
$$

Thus setting $m_{ \pm}=\mu v^{ \pm}$, we see that $m_{+}=m_{-} v$ and $m_{ \pm}-f \in \partial C\left(L^{p}\right)$. In particular $\mu \in \mathrm{L}^{\mathfrak{p}}$ solves $\left(1-\mathrm{C}_{\omega}\right) \mu=0$ iff $\mathrm{m}_{ \pm}=\mu v^{ \pm}$solves the homogeneous RHP.

$$
\mathrm{m}_{+}=\mathrm{m}_{-} v, \quad \mathrm{~m}_{ \pm} \in \partial \mathrm{C}\left(\mathrm{L}^{\mathrm{p}}\right) .
$$

We summarize the above calculations as follows:

Proposition 3.10. Let $1<p<\infty$. Then

$$
\begin{aligned}
\left(1-\mathrm{C}_{\omega}\right) & \text { is a bijection in } \mathrm{L}^{\mathrm{p}}(\Sigma) \\
& \Longleftrightarrow \\
\mathrm{IRHP} 1_{\mathfrak{p}} & \text { has a unique solution for all } \mathrm{f} \in \mathrm{L}^{\mathrm{p}}(\Sigma) \\
& \Longleftrightarrow \\
\mathrm{IRHP} 2_{\mathrm{p}} & \text { has a unique solution for all } \mathrm{F} \in \mathrm{L}^{\mathrm{p}}(\Sigma) .
\end{aligned}
$$

Moreover, if one, and hence all three of the above conditions, is satisfied, then for all $f \in \mathrm{L}^{\mathrm{p}}(\Sigma)$

$$
\begin{aligned}
\left(1-C_{\omega}\right)^{-1} f & =m_{+}\left(v^{+}\right)^{-1}=m_{-}\left(v^{-}\right)^{-1} \\
& =\left(M_{+}+f\right)\left(v^{+}\right)^{-1}=\left(M_{-}+f\right)\left(v^{-}\right)^{-1}
\end{aligned}
$$

where $\mathrm{m}_{ \pm}$solves IRHP $1_{\mathrm{p}}$ with the given $\mathrm{f}$ and $\mathrm{M}_{ \pm}$solves IRHP $2_{\mathrm{p}}$ with $\mathrm{F}=\mathrm{f}(v-\mathrm{I})$ $\left(\in \mathrm{L}^{p} !\right)$, and if $\mathrm{M}_{ \pm}$solves IRHP2 $p$ with $\mathrm{F} \in \mathrm{L}^{\mathrm{p}}(\Sigma)$, then

$$
\begin{aligned}
& M_{+}=\left(\left(1-C_{\omega}\right)^{-1}\left(C^{-} F\right)\right) v^{+}+F \text { and } \\
& M_{-}=\left(\left(1-C_{\omega}\right)^{-1}\left(C^{-} F\right)\right) v^{-} .
\end{aligned}
$$

Finally, if $\mathrm{f} \in \mathrm{L}^{\infty}(\Sigma)$ and $v^{ \pm}-\mathrm{I} \in \mathrm{L}^{\mathrm{p}}(\Sigma)$, then (3.11) remains valid provided we interpret

$$
\left(1-C_{\omega}\right)^{-1} f \equiv f+\left(1-C_{\omega}\right)^{-1} C_{\omega} f .
$$

This is true, in particular, for the normalized $\operatorname{RHP}(\Sigma, v)_{p}$ where $f \equiv I$.

Remark. If $1-C_{\omega}$ is invertible, for one choice of $v^{ \pm}$, then (exercise) it is invertible for all choices of $v^{ \pm}$such that

$$
v=\left(v^{-}\right)^{-1} v^{+}, \quad v^{ \pm}, \quad\left(v^{ \pm}\right)^{-1} \in \mathrm{L}^{\infty}(\Sigma) .
$$

Note that if we take $v^{+}=v, v^{-}=\mathrm{I}$, in particular, then

$$
\mathrm{C}_{\omega} \mathrm{h}=\mathrm{C}^{-}(\mathrm{h}(v-\mathrm{I})) \text {. }
$$


The above Proposition implies, in particular, that if $\mu \in \mathrm{I}+\mathrm{L}^{p}$ solves

$$
\left(1-\mathrm{C}_{\omega}\right) \mu=\mathrm{I}
$$

in the sense of (3.12) i.e. $\mu=I+v, v \in L^{p}$

$$
\left(1-\mathrm{C}_{\omega}\right) v=\mathrm{C}_{\omega} \mathrm{I}=\mathrm{C}^{+} \omega_{-}+\mathrm{C}^{-} \omega_{+} \in \mathrm{L}^{\mathrm{p}}
$$

then $\mathrm{m}_{ \pm}=\mu v^{ \pm}$solves the normalized $\operatorname{RHP}(\Sigma, v)_{\mathrm{p}}$. It is in this precise sense that the solution of the normalized RHP is equivalent to the solution of a singular integral equation (3.13), (3.14) on $\Sigma$.

One very important consequence of the proof of Proposition 3.10 is given by the following

Corollary 3.15. Let $f \in \mathrm{L}^{\mathrm{p}}(\Sigma)$.

Let $\mathrm{m}_{ \pm}$solve IRHP1 $1_{\mathrm{p}}$ with the given $\mathrm{f}$ and let $\mathrm{M}_{ \pm}$solve IRHP2 $\mathrm{p}$ with $\mathrm{F}=\mathrm{f}(v-\mathrm{I})$. Then

$$
\left\|\left(1-C_{\omega}\right)^{-1} f\right\|_{p} \leqslant c\left\|m_{ \pm}\right\|_{p}
$$

and

$$
\left\|\left(1-C_{\omega}\right)^{-1} f\right\|_{p} \leqslant c^{\prime}\left(\left\|M_{ \pm}\right\|_{p}+\|f\|_{p}\right)
$$

for some constants $\mathrm{c}=\mathrm{c}_{\mathrm{p}}, \mathrm{c}^{\prime}=\mathrm{c}_{\mathrm{p}}^{\prime}$. In particular if we know, or can show, that $\left\|\mathrm{m}_{ \pm}\right\|_{\mathrm{p}} \leqslant$ const $\|\mathrm{f}\|_{\mathrm{p}}$, or $\left\|\mathrm{M}_{ \pm}\right\|_{\mathrm{p}} \leqslant$ const $\|\mathrm{f}\|_{\mathrm{p}}$, then we can conclude from (3.16) or (3.17) that $\left(1-\mathrm{C}_{\omega}\right)^{-1}$ is bounded in $\mathrm{L}^{\mathrm{p}}$ with a corresponding bound. Conversely if we know that $\left(1-\mathrm{C}_{\omega}\right)^{-1}$ exists, then the above calculations show that $\left\|\mathrm{m}_{ \pm}\right\|_{\mathrm{p}} \leqslant \tilde{\mathrm{c}}\|\mathrm{f}\|_{\mathrm{p}}$ and $\left\|M_{ \pm}\right\|_{p} \leqslant \tilde{\tilde{c}}\|f\|_{p}$ for corresponding constants $\tilde{\mathbf{c}}, \tilde{\tilde{c}}$.

Finally we consider uniqueness for the solution of the normalized $\operatorname{RHP}(\Sigma, v)_{p}$ as given in Definition 3.4. Observe first that if $F(z)=(C f)(z)$ for $f \in L^{p}(\Sigma)$ and $\mathrm{G}(z)=(\mathrm{Cg})(z)$ for $\mathrm{g} \in \mathrm{Lq}(\Sigma), \frac{1}{\mathrm{r}}=\frac{1}{\mathrm{p}}+\frac{1}{\mathrm{q}} \leqslant 1,1<\mathrm{p}, \mathrm{q}<\infty$, then a simple computation shows that

$$
\mathrm{FG}(z)=\operatorname{Ch}(z)
$$

where

$$
h(s)=-\frac{1}{2 i}(g(s)(H f)(s)+f(s)(H g)(s))
$$

where again $\mathrm{Hf}(\mathrm{s})=$ Hilbert transform $=\lim _{\epsilon \downarrow 0} \int_{\left|\mathrm{s}^{\prime}-\mathrm{s}\right|>\epsilon} \frac{\mathrm{f}\left(\mathrm{s}^{\prime}\right)}{\mathrm{s}-\mathrm{s}^{\prime}} \frac{\mathrm{ds}^{\prime}}{\pi}$, and similarly for $\mathrm{Hg}(\mathrm{s})$. As $\mathrm{h}$ clearly lies in $\mathrm{L}^{\mathrm{r}}(\Sigma), \mathrm{r} \geqslant 1$, it follows that

$$
\mathrm{F}_{+} \mathrm{G}_{+}(z)-\mathrm{F}_{-} \mathrm{G}_{-}(z)=h(z) \quad \text { for a.e. } \quad z \in \Sigma .
$$

(Note: $C^{+} h(z)-C^{-} h(z)=h(z)$ even if $h$ is in $L^{1}$, even though $C^{ \pm}$is not bounded in $\mathrm{L}^{1}$.)

Theorem 3.20. Fix $1<p<\infty$. Suppose $m_{ \pm}$solves the normalized RHP $(\Sigma, v)_{p}$. Suppose that $\mathrm{m}_{ \pm}^{-1}$ exists a.e. on $\Sigma$ and $\mathrm{m}_{ \pm}^{-1} \in \mathrm{I}+\partial \mathrm{C}\left(\mathrm{L}^{\mathrm{q}}\right), 1<\mathrm{q}<\infty, \frac{1}{\mathrm{r}}=\frac{1}{\mathrm{p}}+\frac{1}{\mathrm{q}} \leqslant 1$. Then the solution of the normalized RHP $(\Sigma, v)_{p}$ is unique. 
Proof. Suppose $\hat{m}_{ \pm}=I+C^{ \pm} \hat{h}, \hat{h} \in L^{p}(\Sigma)$ is a $2^{\text {nd }}$ solution of the normalized RHP. We have, by assumption, $\mathrm{m}_{ \pm}^{-1}=\mathrm{I}+\mathrm{C}^{ \pm} \mathrm{k}$ for some $\mathrm{k} \in \mathrm{L}^{\mathrm{q}}(\Sigma)$. (It is an Exercise to show that $\mathrm{I}+(\mathrm{Ck})(z)$, the extension of $\mathrm{m}_{ \pm}^{-1}$ to $\mathbb{C} \backslash \Sigma$, is in fact $\mathrm{m}(z)^{-1}$.).

Then arguing as above

$$
\begin{aligned}
\hat{\mathrm{m}}_{ \pm} \mathrm{m}_{ \pm}^{-1}-\mathrm{I} & =\left(\hat{\mathrm{m}}_{ \pm}-\mathrm{I}\right)\left(\mathrm{m}_{ \pm}^{-1}-\mathrm{I}\right)+\left(\hat{\mathrm{m}}_{ \pm}-\mathrm{I}\right)+\left(\mathrm{m}_{ \pm}^{-1}-\mathrm{I}\right) \\
& =\mathrm{C}^{ \pm} \mathrm{h}
\end{aligned}
$$

for some $h \in \mathrm{L}^{\mathrm{r}}(\Sigma)+\mathrm{L}^{\mathrm{p}}(\Sigma)+\mathrm{L}^{\mathrm{q}}(\Sigma)$.

Hence

$$
\hat{m}_{+} \mathrm{m}_{+}^{-1}-\hat{m}_{-} \mathrm{m}_{-}^{-1}=\mathrm{h} .
$$

But

$$
\hat{\mathrm{m}}_{+} \mathrm{m}_{+}^{-1}=\left(\hat{\mathrm{m}}_{-} v\right)\left(\mathrm{m}_{-} v\right)^{-1}=\hat{\mathrm{m}}_{-} \mathrm{m}_{-}^{-1}
$$

and so $h=0$. Thus $\hat{m}_{ \pm} m_{ \pm}^{-1}-I=0$ or $\hat{m}_{ \pm}=m_{ \pm}$.

Theorem 3.21. If $\mathrm{n}=2, \mathrm{p}=2$ and $\operatorname{det} v(z)=1$ a.e. on $\Sigma$, then the solution of the normalized RHP $(\Sigma, v)_{2}$ is unique.

Proof. Because $n=2$ and $p=2,(3.18),(3.19) \Longrightarrow(\operatorname{det} m(z))_{ \pm}=1+C^{ \pm} h$, where $h \in \mathrm{L}^{1}(\Sigma)+\mathrm{L}^{2}(\Sigma)$ and so $(\operatorname{det} \mathrm{m})_{+}-(\operatorname{det} \mathrm{m})_{-}=\mathrm{h}(z)$ a.e. But $\operatorname{det} \mathrm{m}_{+}=\operatorname{det} \mathrm{m}_{-}$ as $\operatorname{det} v=1$, and so $h \equiv 0$. But then $\operatorname{det} m(z)_{ \pm}=1$. Hence, if

$$
m_{ \pm}=\left(\begin{array}{ll}
m_{11 \pm} & m_{12 \pm} \\
m_{21 \pm} & m_{22 \pm}
\end{array}\right)
$$

we have

$$
m_{ \pm}^{-1}=\left(\begin{array}{cc}
m_{22} & -m_{12 \pm} \\
-m_{21 \pm} & m_{11 \pm}
\end{array}\right)
$$

and so clearly $m_{ \pm}^{-1} \in I+\partial C\left(L^{2}\right)$. The result now follows from Theorem 3.20.

These results immediately imply that the normalized RHP $\left(\Sigma=\mathbb{R}, v_{x, t}\right)$ for MKdV with $v_{x, t}$ given by (1.23) has a unique solution in $\mathrm{L}^{2}(\mathbb{R})$. Indeed, factorize

$$
\begin{aligned}
v_{x, \mathrm{t}}(z)=\left(v_{x, \mathrm{t}}^{-}\right)^{-1} v_{x, \mathrm{t}}^{+} & =\left(\mathrm{I}-w_{x, \mathrm{t}}^{-}\right)^{-1}\left(\mathrm{I}+w_{x, \mathrm{t}}^{+}\right) \\
& =\left(\begin{array}{cc}
1 & -\overline{\mathrm{r}} e^{-2 i \tau} \\
0 & 1
\end{array}\right)\left(\begin{array}{cc}
1 & 0 \\
r e^{2 i \tau} & 1
\end{array}\right)
\end{aligned}
$$

so that

$$
w_{x, \mathrm{t}}=\left(w_{x, \mathrm{t}}^{-}, w_{x, \mathrm{t}}^{+}\right)=\left(\left(\begin{array}{cc}
0 & -\overline{\mathrm{r}} e^{-2 i \tau} \\
0 & 0
\end{array}\right),\left(\begin{array}{cc}
0 & 0 \\
r \mathrm{e}^{2 i \tau} & 0
\end{array}\right)\right)
$$

But for $\Sigma=\mathbb{R}$, we have

Exercise 3.22. Both $\mathrm{C}^{+}$and $-\mathrm{C}^{-}$are orthogonal projections in $\mathrm{L}^{2}(\mathbb{R})$ and so $\left\|\mathrm{C}^{ \pm}\right\|_{\mathrm{L}^{2}}=1$. 
Using the Hilbert-Schmidt matrix norm $\|M\|=\left(\sum_{i, j}\left|M_{i j}\right|^{2}\right)^{\frac{1}{2}}$, we have

$$
\begin{aligned}
\left\|C_{\omega_{x, t}} h\right\|_{L^{2}}^{2}= & \| C^{+}\left(\begin{array}{ll}
h_{11} & h_{12} \\
h_{21} & h_{22}
\end{array}\right)\left(\begin{array}{cc}
0 & -\bar{r} e^{-2 i \tau} \\
0 & 0
\end{array}\right) \\
& \quad+C^{-}\left(\left(\begin{array}{ll}
h_{11} & h_{12} \\
h_{21} & h_{22}
\end{array}\right)\left(\begin{array}{cc}
0 & 0 \\
r e^{2 i \tau} & 0
\end{array}\right) \|_{L^{2}}^{2}\right. \\
= & \|\left(\begin{array}{cc}
0 & C^{+} h_{11}(-\bar{r}) e^{2 i \tau} \\
0 & C^{+} h_{21}(-\bar{r}) e^{-2 i \tau}
\end{array}\right) \\
\quad & \quad+\left(\begin{array}{cc}
C^{-} h_{12} r e^{2 i \tau} & 0 \\
C^{-} h_{22} r e^{2 i \tau} & 0
\end{array}\right) \|^{2} \\
= & \left\|C^{-} h_{12} r e^{2 i \tau}\right\|_{L^{2}}^{2}+\left\|C^{-} h_{22} r e^{2 i \tau}\right\|_{L^{2}}^{2} \\
& +\left\|C^{+} h_{11}(-\bar{r}) e^{-2 i \tau}\right\|_{L^{2}}^{2}+\left\|C^{+} h_{21}(-\bar{r}) e^{-2 i \tau}\right\|_{L^{2}}^{2} \\
\leqslant & \|r\|_{\infty}^{2}\left(\left\|h_{12}\right\|_{L^{2}}^{2}+\left\|h_{22}\right\|_{L^{2}}^{2}+\left\|h_{11}\right\|_{L^{2}}^{2}+\left\|h_{21}\right\|_{L^{2}}^{2}\right) \\
= & \left\|r_{\infty}\right\|^{2}\|h\|_{L^{2}}^{2}
\end{aligned}
$$

and so, as $\|r\|_{\infty}<1$,

$$
\left\|\mathrm{C}_{\omega_{\mathrm{x}, \mathrm{t}}}\right\|<1 \text {. }
$$

It follows that for each $x, t \in \mathbb{R},\left(1-\mathrm{C}_{\omega_{x, t}}\right)^{-1}$ exists in $\mathrm{L}^{2}(\mathbb{R})$ and

$$
\left\|\left(1-\mathrm{C}_{\omega_{\mathrm{x}, \mathrm{t}}}\right)^{-1}\right\|_{\mathrm{L}^{2}} \leqslant \frac{1}{1-\|\mathrm{r}\|_{\infty}}<\infty
$$

and the proof of the existence and uniqueness for $\left(\Sigma, v_{x, t}\right)$ follows from Proposition 3.10. On the other hand, just uniqueness alone follows from Theorem 3.21 as $\operatorname{det} v(z) \equiv 1$ on $\mathbb{R}$.

Now it turns out that a key role in the theory of RHPs is played by Fredholm operators. Recall that a bounded linear operator $\mathrm{T}$ from a Banach space $\mathrm{X}$ to a Banach space $Y$ is Fredholm if

$$
\operatorname{dim} \operatorname{ker} T<\infty
$$

and

$$
\operatorname{dim} \text { coker } \mathrm{T}<\infty \quad \text { i.e. } \mathrm{Y} / \mathrm{ran} \mathrm{T} \text { is a finite dimensional space. }
$$

If $\mathrm{T}$ is Fredholm, we define index $\mathrm{T} \equiv \operatorname{dim} \operatorname{ker} \mathrm{T}-\operatorname{dim} \operatorname{coker} \mathrm{T}$.

Exercise 3.23. If $T: X \rightarrow Y$ is Fredholm, then ran $T$ is closed in $Y$.

Exercise 3.24. $\mathrm{T}: \mathrm{X} \rightarrow \mathrm{Y}$ is Fredholm iff it has a pseudo-inverse $\mathrm{S} \in \mathcal{L}(\mathrm{Y}, \mathrm{X})$ such that $\mathrm{ST}=1_{X}+\mathrm{K}$ and $\mathrm{TS}=1_{Y}+\mathrm{L}$ where $\mathrm{K}$ is a compact operator in $\mathcal{L}(\mathrm{X})$ and $\mathrm{L}$ is a compact operator in $\mathcal{L}(Y)$. 
We know that a normalized RHP $(\Sigma, v)_{\mathrm{p}}$, say, has a (unique) solution if $(1-$ $\left.\mathrm{C}_{\omega}\right)^{-1}$ exists. The situation where we know, for example, that $\left\|C_{\omega}\right\|_{L^{2}}<1$, as in the example $\left(\Sigma=\mathbb{R}, v_{x, t}\right)$ above so that $\left(1-C_{\omega}\right)^{-1}$ exists, is very rare. For example, for the $\mathrm{KdV}$ equation on $\mathbb{R}$

$$
\begin{aligned}
& u_{t}+6 u u_{x}+u_{x x x}=0, \\
& u(x, 0)=u_{0}(x) \rightarrow 0 \quad \text { as } \quad|x| \rightarrow \infty,
\end{aligned}
$$

the associated RHP is exactly the same as $\left(\mathbb{R}, v_{x, t}\right)$ for $M K d V$, except that now, generically,

$$
|r(z)|<1 \quad \text { for } \quad|z|>0
$$

but

$$
|r(0)|=1
$$

Thus $\|r\|_{\infty}=1$ and the above proof of the existence and uniqueness for the RHP breaks down. A more general approach to proving the existence and uniqueness of solutions to normalized RHPs, is to attempt the following:

- Prove $1-\mathrm{C}_{\omega}$ is Fredholm.

- Prove ind $\left(1-\mathrm{C}_{\omega}\right)=0$.

- Prove dim $\operatorname{ker}\left(1-\mathrm{C}_{\omega}\right)=0$.

Then it follows that $1-\mathrm{C}_{\omega}$ is a bijection, and hence the normalized $\operatorname{RHP}(\Sigma, v)$ has a unique solution.

Let's see how this goes for KdV with normalized RHP $\left(\Sigma=\mathbb{R}, v_{x, t}\right)$, but now $r$ satisfies (3.25), (3.26). By our previous comments (see Remark above), it is enough to consider the special case $v^{+}=v, v^{-}=\mathrm{I}$ so that $\omega^{+}=v-\mathrm{I}$ and $\omega^{-}=0$. Thus

$$
\mathrm{C}_{\omega} \mathrm{h}=\mathrm{C}^{-} \mathrm{h}(v-\mathrm{I}) .
$$

We assume $r(z)$ is continuous and $r(z) \rightarrow 0$ as $|z| \rightarrow \infty$. Let $S$ be the operator

$$
\mathrm{Sh}=\mathrm{C}^{-} \mathrm{h}\left(v^{-1}-\mathrm{I}\right) \text {. }
$$

Then

$$
\begin{aligned}
& \mathrm{C}_{\omega} \mathrm{Sh}=\mathrm{C}^{-}(\mathrm{Sh}(v-\mathrm{I})) \\
&= \mathrm{C}^{-}\left[\left(\mathrm{C}^{-} \mathrm{h}\left(v^{-1}-\mathrm{I}\right)\right)(v-\mathrm{I})\right] \\
&= \mathrm{C}^{-}\left[\left(\mathrm{C}^{+} \mathrm{h}\left(v^{-1}-\mathrm{I}\right)\right)(v-\mathrm{I})\right] \\
&-\mathrm{C}^{-}\left[\mathrm{h}\left(v^{-1}-\mathrm{I}\right)(v-\mathrm{I})\right] \\
& \text { as } \mathrm{C}^{+}-\mathrm{C}^{-}=1 . \text { But h }\left(v^{-1}-\mathrm{I}\right)(v-\mathrm{I})=\mathrm{h}\left(2 \mathrm{I}-v-v^{-1}\right)=\mathrm{h}(\mathrm{I}-v)+\mathrm{h}\left(\mathrm{I}-v^{-1}\right) . \\
& \text { Thus } \quad \\
& \mathrm{C}_{\omega} \mathrm{Sh}=\mathrm{C}^{-}\left[\left(\mathrm{C}^{+} \mathrm{h}\left(v^{-1}-\mathrm{I}\right)\right)(v-\mathrm{I})\right] \\
&+\mathrm{C}^{-}\left(\mathrm{h}\left(v^{-1}-\mathrm{I}\right)\right)+\mathrm{C}^{-}(\mathrm{h}(v-\mathrm{I}))
\end{aligned}
$$




$$
=\mathrm{C}^{-}\left[\left(\mathrm{C}^{+} \mathrm{h}\left(v^{-1}-\mathrm{I}\right)\right)(v-\mathrm{I})\right]+\mathrm{C}_{\omega} \mathrm{h}+\mathrm{Sh}
$$

and we see that

$$
\left(1-C_{\omega}\right)(1-S) h=h+C^{-}\left[\left(C^{+} h\left(v^{-1}-I\right)\right)(v-I)\right] .
$$

But

Exercise 3.27. $\quad \mathrm{Kh}=\mathrm{C}^{-}\left[\left(\mathrm{C}^{+} \mathrm{h}\left(v^{-1}-\mathrm{I}\right)\right)(v-\mathrm{I})\right] \quad$ is compact in $\mathrm{L}^{2}(\mathbb{R})$.

Hint: $v-\mathrm{I}$ is a continuous function which $\rightarrow 0$ as $|z| \rightarrow \infty$ and hence can be approximated in $L^{\infty}(\mathbb{R})$ by finite linear combinations of functions of the form $a /\left(z-z^{\prime}\right)$ for suitable constants $a$ and points $z^{\prime} \in \mathbb{C} \backslash \mathbb{R}$. Then use the following fact:

Exercise 3.28. If $T_{n}, n \geqslant 1$ are compact operators in $\mathcal{L}(X, Y)$ and $\left\|T_{n}-T\right\| \rightarrow 0$ as $n \rightarrow \infty$ for some operator $T \in \mathcal{L}(X, Y)$, then $T$ is compact.

Similarly

$$
(1-\mathrm{S})\left(1-\mathrm{C}_{\omega}\right)=1+\mathrm{L}, \quad \text { L compact. }
$$

Thus $\left(1-\mathrm{C}_{\omega}\right)$ is Fredholm.

Now we use the following fact:

Exercise 3.29. Suppose that for $\gamma \in[0,1], \mathrm{T}(\gamma)$ is a norm-continuous family of Fredholm operators. Then for $\gamma \in[0,1]$,

$$
\operatorname{ind} \mathrm{T}(\gamma)=\mathrm{const}=\operatorname{ind} \mathrm{T}(0)=\operatorname{ind} \mathrm{T}(1) \text {. }
$$

Apply this fact to $C_{\omega(\gamma)}$, where we replace $r$ by $\gamma r$ in $v_{x, t}$,

$$
v_{x, t, \gamma}=\left(\begin{array}{cc}
1-\gamma^{2}|r|^{2} & -\gamma \bar{r} e^{-2 i \tau} \\
\gamma r e^{2 i \tau} & 1
\end{array}\right) .
$$

The proof above shows that $\mathrm{C}_{\omega(\gamma)}$ is a norm continuous family of Fredholm operators and so ind $\left(1-C_{\omega}\right)=\operatorname{ind}\left(1-C_{\omega(\gamma=1)}\right)=\operatorname{ind}\left(1-C_{\omega(\gamma=0)}\right)=0$ as $\mathrm{C}_{\omega(\gamma=0)}=0$ and the index of the identity operator is clearly 0 .

Finally suppose

$$
\left(1-\mathrm{C}_{\omega}\right) \mu=0 .
$$

Then using (3.9), $m_{+}=\mu v$ and $m_{-}=\mu$ solve $m_{+}=m_{-} v, m_{ \pm} \in \partial C\left(L^{2}\right)$.

Consider $\mathrm{P}(z)=\mathrm{m}(z)(\mathrm{m}(\bar{z}))^{*}$ for $z \in \mathbb{C}^{+}$where $\mathrm{m}(z)$ is the extension of $\mathrm{m}_{ \pm}$ off $\mathbb{R}$ i.e. if $m_{ \pm}=C^{ \pm} h, h \in L^{2}$, then $m(z)=(C h)(z)$. Then for a contour $\Gamma_{R, \epsilon}$, pictured in Figure 3.30, $\int_{\Gamma_{R, \epsilon}} \mathrm{P}(z) \mathrm{d} z=0$ as $\mathrm{P}(z)$ is analytic.

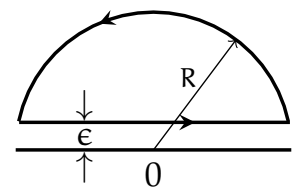

Figure 3.30. A semi-circle $\epsilon$ above the real axis. 
Letting $\epsilon \downarrow 0$ and $R \rightarrow \infty$, we obtain (exercise) $\int_{-\infty}^{\infty} P_{+}(z) d z=0$; i.e.

$$
0=\int_{\mathbb{R}} m_{+}(z) m_{-}(z)^{*} d z=\int_{\mathbb{R}} m_{-}(z) v(z) m_{-}(z)^{*} d z .
$$

Taking adjoints and adding, we find

$$
0=\int_{\mathbb{R}} \mathrm{m}_{-}(z)\left(v+v^{*}\right)(z) \mathrm{m}_{-}(z)^{*} \mathrm{~d} z .
$$

But a direct calculation shows that $v+v^{*}$ is diagonal and

$$
\left(v+v^{*}\right)(z)=2\left(\begin{array}{cc}
1-|r(z)|^{2} & 0 \\
0 & 1
\end{array}\right) .
$$

Now since $|r(z)|<1$ a.e. (in fact everywhere except $z=0$ ), we conclude that $\mathrm{m}_{-}(z)=0$. But $\mu=\mathrm{m}_{-}$and so we see that $\operatorname{ker}\left(1-\mathrm{C}_{\boldsymbol{\omega}}\right)=\{0\}$.

The result of the above chain of arguments is that the solution of the normalized RHP $\left(\Sigma, v_{x, t}\right)$ for KdV exists and is unique. Such Fredholm arguments have wide applicability in Riemann-Hilbert Theory [22].

One last general remark. The scalar case $n=1$ is special. This is because the RHP can be solved explicitly by formula. Indeed, if $m_{+}=m_{-} v$, then it follows that $(\log m)_{+}=\left(\log m_{-}\right)+\log v$ and hence $\log m(z)$ is given by Plemelj's formula, which provides the general solution of additive RHPs, via

and so

$$
\log \mathrm{m}=\mathrm{C}(\log v)(z)=\int_{\Sigma} \frac{\log v(\mathrm{~s})}{\mathrm{s}-z} \mathrm{ds}
$$

$$
\mathrm{m}(z)=\exp \left(\int_{\Sigma} \frac{\log v(\mathrm{~s})}{\mathrm{s}-z} \mathrm{ds}\right)
$$

a formula which is easily checked directly. However, there is a hidden subtlety in the business: On $\mathbb{R}$, say, although $v(s)$ may go rapidly to 0 as $s \rightarrow \pm \infty, v(s)$ may wind around 0 and so $\log v(s)$ may not be integrable at both $\pm \infty$. Thus there is a topological obstacle to the existence of a solution of the RHP. If $n>1$, there are many more such "hidden" obstacles.

\section{Lecture 4}

RHP's arise in many difference ways. For example, consider orthogonal polynomials: we are given a measure $\mu$ on $\mathbb{R}$ with finite moments,

$$
\int_{\mathbb{R}}|x|^{\mathrm{m}} \mathrm{d} \mu(x)<\infty \quad \text { for } \quad \mathrm{m}=0,1,2, \ldots
$$

Performing Gram-Schmidt on $1, x, x^{2}, \ldots$ with respect to $d \mu(x)$, we obtain (monic) orthogonal polynomials

$$
\pi_{n}(x)=x^{n}+\ldots, \quad, n \geqslant 0
$$

such that

$$
\int_{\mathbb{R}} \pi_{n}(x) \pi_{m}(x) d \mu u(x)=0, \quad n \neq m, \quad n, m \geqslant 0 .
$$


(Here we assume that $d \mu$ has infinite support: otherwise there are only a finite number of such polynomials.)

Associated with the $\pi_{\mathrm{n}}$ 's are the orthonormal polynomials

$$
P_{n}(x)=\gamma_{n} \pi_{n}(x), \quad \gamma_{n}>0, \quad n \geqslant 0
$$

such that

$$
\int_{\mathbb{R}} P_{n}(x) P_{m}(x) d \mu(x)=\delta_{n, m}, \quad n, m \geqslant 0 .
$$

Orthogonal polynomials are of great historical and continuing importance in many different areas of mathematics, from algebra, through combinatorics, to analysis. The classical orthogonal polynomials, such as the Hermite polynomials, the Legendre polynomials, the Krawchouk polynomials, are well known and much is known about their properties. In view of our earlier comments it should come as no surprise that much of this knowledge, particularly asymptotic properties, follows from the fact that these polynomials have integral representations analogous to the integral representation for the Airy function in the first lecture. For example, for the Hermite polynomials

one has the integral representation

$$
\int_{\mathbb{R}} H_{n}(x) H_{m}(x) e^{-x^{2}} d x=0 \quad n \neq m, \quad n, m \geqslant 0
$$

$$
H_{n}(x)=n ! \int_{\mathcal{C}} \omega^{-n-1} e^{2 x \omega-\omega^{2}} d \omega
$$

where $\mathcal{C}$ is a (small) circle enclosing the origin, (Note: the $\mathrm{H}_{n}$ 's are not monic, but are proportional to the $\pi_{n}{ }^{\prime} s, H_{n}(x)=c_{n} \pi_{n}(x)$ where the $c_{n}$ 's are explicit) and the asymptotic behavior of the $\mathrm{H}_{n}$ 's follow from the classical steepest descent method. For general weights, however, no such integral representations are known.

The Hermite polynomials play a key role in random matrix theory in the socalled Gaussian Unitary, Orthogonal and Symplectic Ensembles. However it was long surmised that local properties of random matrix ensembles were universal, i.e., independent of the underlying weights. In other words if one considers general weights such as $e^{-x^{4}} d x, e^{-\left(x^{6}+x^{4}\right)} d x$, etc., instead of the weight $e^{-x^{2}} d x$ for the Hermite polynomials, the local properties of the random matrices, at the technical level, boil down to analyzing the asymptotics of the polynomials orthogonal with respect to the weights $e^{-x^{4}} d x, e^{-\left(x^{6}+x^{4}\right)} d x$, etc., for which no integral representations are known. What to do?

It turns out however, that orthogonal polynomials with respect to an arbitrary weight can be expressed in terms of a RHP. Suppose $d \mu(x)=\omega(x) d x$, for some $\omega(x) \geqslant 0$ such that

$$
\int_{\mathbb{R}}|x|^{\mathrm{m}} \omega(x) \mathrm{d} x<\infty, \quad \mathrm{m}=0,1,2, \ldots
$$

and suppose for simplicity that

$$
\omega \in \mathrm{H}^{1}(\mathbb{R})=\left\{f \in \mathrm{L}^{2}: \mathrm{f}^{\prime} \in \mathrm{L}^{2}\right\} .
$$


Fix $n \geqslant 0$ and let $Y^{(n)}=\left\{Y_{i j}^{(n)}(z)\right\}_{1 \leqslant i, j \leqslant 2}$ solve the $\operatorname{RHP}\left(\Sigma=\mathbb{R}, v=\left(\begin{array}{cc}1 & w \\ 0 & 1\end{array}\right)\right)$ normalized so that

$$
Y^{(n)}(z)\left(\begin{array}{cc}
z^{-n} & 0 \\
0 & z^{n}
\end{array}\right) \rightarrow I \quad \text { as } \quad z \rightarrow \infty .
$$

Exercise 4.3. Show that we then have (see e.g. [12])

$$
Y^{(n)}(z)=\left(\begin{array}{cc}
\pi_{n}(z) & C\left(\pi_{n} \omega\right) \\
-2 \pi i \gamma_{n-1}^{2} \pi_{n-1}(z) & C\left(-2 \pi i \gamma_{n-1}^{2} \pi_{n-1} \omega\right)
\end{array}\right)
$$

where $C=C_{\mathbb{R}}$ is the Cauchy operator on $\mathbb{R}, \pi_{n}, \pi_{n-1}$ are the monic orthogonal polynomials with respect to $\omega(x) d x$ and $\gamma_{n-1}$ is the normalization coefficient for $\pi_{n-1}$ as in (4.1). (Note that by (4.2) and Theorem 2.15, $Y^{(n)}(z)$ is continuous down to the axis for all z.) This discovery is due to Fokas, Its and Kitaev [21]. Moreover this is just exactly the kind of problem to which the nonlinear steepest descent method can be applied to obtain $([14,15])$ the asymptotics of the $\pi_{\mathrm{n}}$ 's with comparable precision to the classical cases, Hermite, Legendre, ..., and so prove universality for unitary ensembles (and later, Deift and Gioev, Shcherbina, for Orthogonal \& Symplectic Ensembles of random matrices, see [13] and the references therein).

As mentioned earlier, RHPs are useful not only for asymptotic analysis, but also to analyze analytical and algebraic issues. Here we show how RHPs give rise to difference equations, or differential equations, in other situations.

Consider the solution $Y^{(n)}$ for the orthogonal polynomial $\operatorname{RHP}\left(\mathbb{R}, v=\left(\begin{array}{ll}1 & \omega \\ 0 & 1\end{array}\right)\right)$. The key fact is that the jump matrix $\left(\begin{array}{cc}1 & w \\ 0 & 1\end{array}\right)$ is independent of $n$ : the dependence on $\mathrm{n}$ is only in the boundary condition

$$
Y^{(n)}\left(\begin{array}{cc}
z^{-n} & 0 \\
0 & z^{+n}
\end{array}\right) \rightarrow I
$$

So we have $Y_{+}^{(n+1)}=Y_{-}^{(n+1)} v$ and $Y_{+}^{(n)}=Y_{-}^{(n)} v$.

$$
\text { Let } \begin{aligned}
& R(z)=Y^{(n+1)}(z)\left(Y^{(n)}(z)\right)^{-1}, z \in \mathbb{C} \backslash \mathbb{R} \text {. Then } \\
& \qquad \begin{aligned}
R_{+}(z) & =Y_{+}^{(n+1)}(z)\left(Y_{+}^{(n)}(z)\right)^{-1} \\
& =\left(Y_{-}^{(n+1)}(z) v(z)\right)\left(Y_{-}^{(n)}(z) v(z)\right)^{-1} \\
& =Y_{-}^{(n+1)}(z)\left(v(z) v(z)^{-1}\right)\left(Y_{-}^{(n)}(z)\right)^{-1} \\
& =R_{-}(z) .
\end{aligned}
\end{aligned}
$$

Hence $R(z)$ has no jump across $\mathbb{R}$ and so, by an application of Morera's Theorem, $\mathrm{R}(z)$ is in fact entire. But as $z \rightarrow \infty$

$$
\mathrm{R}(z)=\left[Y^{(\mathrm{n}+1)}(z)\left(\begin{array}{cc}
z^{-n-1} & 0 \\
0 & z^{n+1}
\end{array}\right)\right]\left(\begin{array}{cc}
z & 0 \\
0 & z^{-1}
\end{array}\right)\left[Y^{(\mathrm{n})}(z)\left(\begin{array}{cc}
z^{-n} & 0 \\
0 & z^{n}
\end{array}\right)\right]^{-1}
$$




$$
\begin{aligned}
& =\left(\mathrm{I}+\mathrm{O}\left(\frac{1}{z}\right)\right)\left(\begin{array}{cc}
z & 0 \\
0 & z^{-1}
\end{array}\right)\left(\mathrm{I}+\mathrm{O}\left(\frac{1}{z}\right)\right) \\
& =\mathrm{O}(z) .
\end{aligned}
$$

Thus $\mathrm{R}(z)$ must be a polynomial of order 1 ,

$$
Y^{(n+1)}(z)\left(Y^{(n)}(z)\right)^{-1}=R(z)=A z+B
$$

for suitable $A$ and $B$, or,

$$
Y^{(n+1)}(z)=(A z+B) Y^{(n)}(z)
$$

which is a difference equation for orthogonal polynomials with respect to a fixed weight.

Exercise 4.5. Make the argument leading to (4.4) rigorous (why does $\left(Y^{(n)}\right)^{-1}$ exist, etc.)

Exercise 4.6. Show that (4.4) implies the familiar three term recurrence relation for orthogonal polynomials $p_{\mathrm{n}}(z)$

$$
b_{n} p_{n+1}(z)+\left(a_{n}-z\right) p_{n}(z)+b_{n-1} p_{n-1}=0, \quad n \geqslant 0
$$

$a_{\mathrm{n}} \in \mathbb{R}, \quad b_{\mathrm{n}}>0 ; \quad b_{-1} \equiv 0$.

Whereas the RHP for orthogonal polynomials comes "out of the blue", there are some systematic methods to produce RHP representations for certain problems of interest. This is true in particular for RHPs associated with ordinary differential equations. For example, consider the ZS-AKNS equation (ZakharovShabat, Ablowitz-Kaup-Newell-Segur)

$$
\left[\partial_{x}-\left(i z \sigma+\left(\begin{array}{cc}
0 & q(x) \\
\bar{q}(x) & 0
\end{array}\right)\right)\right] \psi=0, \quad-\infty<x<\infty
$$

(see e.g. [17]). Here $z \in \mathbb{C}, \quad \sigma=\frac{1}{2}\left(\begin{array}{cc}1 & 0 \\ 0 & -1\end{array}\right)$ and $q(x) \rightarrow 0$ at some sufficiently fast rate as $|x| \rightarrow \infty$. Equation (4.7) is intimately connected with the defocusing Nonlinear Schrödinger Equation (NLS) by virtue of the fact that the operator

$$
L=(i \sigma)^{-1}\left(\partial_{x}-\left(\begin{array}{ll}
0 & q \\
\bar{q} & 0
\end{array}\right)\right)
$$

undergoes an isospectral deformation if $\mathrm{q}=\mathrm{q}(\mathrm{t})=\mathrm{q}(\mathrm{x}, \mathrm{t})$ solves NLS

$$
\begin{array}{r}
i q_{t}+q_{x x}-2|q|^{2} q=0 \\
q(x, t=0)=q_{0}(x) .
\end{array}
$$

In other words, if $q=q(t)$ solves NLS then the spectrum of

$$
L(t)=(i \sigma)^{-1}\left(\partial_{x}-\left(\frac{0}{q(x, t)} \begin{array}{c}
q(x, t) \\
0
\end{array}\right)\right)
$$

is constant: Thus the spectrum of $\mathrm{L}(\mathrm{t})$ provides constants of the motion for (4.9), and so NLS is "integrable". The key fact is that there is a RHP naturally associated with L which expresses the integrability of NLS in a form that is useful for 
analysis. Here we follow Beals and Coifman, see [4]. Let $q(x)$ in (4.8) be given with $\mathrm{q}(\mathrm{x}) \rightarrow 0$ as $|x| \rightarrow \infty$ sufficiently rapidly. Then for any $z \in \mathbb{C} \backslash \mathbb{R}$,

Exercise 4.10. The equation $(\mathrm{L}-z) \psi=0$ has a unique solution $\psi$ such that $\psi(x, z) e^{-i x z \sigma} \rightarrow \mathrm{I}$ as $x \rightarrow-\infty$ and is bounded $x \rightarrow \infty$. Such $\psi(x, z)$ are called Beals-Coifman solutions.

Remark 4.11. These solutions have the following properties:

(1) For fixed $x, \psi(x, z)$ is analytic in $\mathbb{C} \backslash \mathbb{R}$, and is continuous down to the axis. That is $\psi_{ \pm}(x, z)=\lim _{\epsilon \downarrow 0} \psi(x, z \pm i \epsilon)$ exist for all $x, z \in \mathbb{R}$.

(2) For fixed $x, \psi(x, z) e^{-i x z \sigma} \rightarrow$ I as $z \rightarrow \infty$,

$$
\psi(x, z) e^{-i x z \sigma}=\mathrm{I}+\frac{\mathrm{m}_{1}(x)}{z}+\mathrm{O}\left(\frac{1}{z^{2}}\right), \quad \text { as } \quad z \rightarrow \infty
$$

for some matrix residue term $m_{1}(x)$.

Now clearly $\psi_{ \pm}(x, z), \quad z \in \mathbb{R}$, are two fundamental solutions of $(L-z) \psi=0$ and so for $z \in \mathbb{R}$,

$$
\psi_{+}(x, z)=\psi_{-}(x, z) v(z)
$$

for all $x \in \mathbb{R}$, where $v(z)$ is independent of $x$. In other words, by (1) of Remark 4.11, $\psi(x, \cdot)$ solves a $\operatorname{RHP}(\Sigma=\mathbb{R}, v)$, normalized as in (4.12). In this way differential equations give rise to RHPs in a systematic way.

One can calculate (exercise) the precise form of $v(z)$ and one finds

$$
v(z)=\left(\begin{array}{cc}
1-|\mathrm{r}(z)|^{2} & \mathrm{r}(z) \\
-\overline{\mathrm{r}(z)} & 1
\end{array}\right), \quad z \in \mathbb{R}
$$

where, again (cf. (1.23) for MKdV) we have for $r$, the reflection coefficient,

$$
\|\mathrm{r}\|_{\infty}<1 \text {. }
$$

Now the map

$$
\mathrm{q} \mapsto \mathrm{r}=\mathcal{R}(\mathbf{q})
$$

is a bijection between suitable spaces: $r=\mathcal{R}(q)$, the direct map, is constructed from $q$ via the solutions $\psi(x, z)$ as above. The inverse map $r \mapsto \mathcal{R}^{-1}(r)=q$ is constructed by solving the RHP $(\Sigma, v)$ normalized by (4.12) for any fixed $x$. One obtains

$$
\psi(x, z) e^{-i z x \sigma}=I+\frac{m_{1}(x ; r)}{z}+O\left(\frac{1}{z^{2}}\right) \quad \text { as } \quad z \rightarrow \infty
$$

and

$$
q(x)=-i\left(m_{1}(x, r)\right)_{12}
$$

(cf (1.24) for MKdV).

Now if $q=q(t)=q(x, t)$ solves NLS then $r(t)=\mathcal{R}(q(t))$ evolves simply,

$$
r(t)=r(t, z)=r(t=0, z) e^{-i t z^{2}}, \quad z \in \mathbb{R}
$$


i.e. $t \rightarrow q(t) \rightarrow r(t) \rightarrow \log r(t)=\log r(t=0)-i t z^{2}$ linearizes NLS. This leads to the following formula for the solution of NLS with initial data $q_{0}$

$$
\mathrm{q}(\mathrm{t})=\mathcal{R}^{-1}\left(e^{-i \mathrm{t}(\cdot)^{2}} \mathcal{R}\left(\mathrm{q}_{0}\right)(\cdot)\right) .
$$

The effectiveness of this representation, which one should view as the RHP analog of NLS of the integral representation (1.2) for the Airy equation, depends on the effectiveness of the nonlinear steepest descent method for RHPs.

Question. Where in the representation (4.14) is the information encoded that $q(t)$ solves NLS?

The answer is as follows. Let $\psi(x, z, t)$ be the solution of the RHP with jump matrix

$$
\nu_{\mathrm{t}}(z)=\left(\begin{array}{cc}
1-|\mathrm{r}|^{2} & \mathrm{r} e^{-i \mathrm{t} z^{2}} \\
-\overline{\mathrm{r}} e^{i \mathrm{t} z^{2}} & 1
\end{array}\right)
$$

normalized as in (4.12). Set $H(x, z, t)=\psi(x, z, t) e^{-i t z^{2} \sigma}$ and observe that

$$
H_{+}=H_{-}\left(\begin{array}{cc}
1-|r|^{2} & r \\
-\bar{r} & 1
\end{array}\right)=H_{-} v
$$

for which the jump matrix is independent of $x$ and $t$. This means that we can differentiate (4.15) with respect to $\mathrm{x}$ and $\mathrm{t}, \mathrm{H}_{\mathrm{x}+}=\mathrm{H}_{\mathrm{x}-} v, \mathrm{H}_{\mathrm{t}+}=\mathrm{H}_{\mathrm{t}-} v$ and conclude, as in the case of orthogonal polynomials, that $\mathrm{H}_{x} \mathrm{H}^{-1}$ and $\mathrm{H}_{\mathrm{t}} \mathrm{H}^{-1}$ are entire, and evaluating these combinations as $z \rightarrow \infty$, we obtain two equations

$$
\mathrm{H}_{\mathrm{x}}=\mathrm{DH} \quad, \mathrm{H}_{\mathrm{t}}=\mathrm{EH}
$$

for suitable polynomials matrix functions $\mathrm{D}$ and $\mathrm{E}$. These functions constitute the famous Lax pair (D, E) for NLS. Compatibility of these two equations requires

$$
\begin{array}{ll} 
& \partial_{t} \partial_{x} H=\partial_{x} \partial_{t} H \\
\Longrightarrow & \partial_{t}(D H)=\partial_{x}(E H) \\
\Longrightarrow & D_{t} H+D E H=E_{x} H+E D H \\
\Longrightarrow & D_{t}+[D, E]=E_{x}
\end{array}
$$

which reduces directly to NLS. In this way RHP's lead to difference and differential equations.

Another systematic way that RHP's arise is through the distinguished class of so-called integrable operators. Let $\Sigma$ be an oriented contour in $\mathbb{C}$ and let $f_{1}, \ldots, f_{n}$ and $g_{1}, \ldots, g_{n}$ be bounded measurable functions on $\Sigma$. We say that an operator $K$ acting on $\mathrm{L}^{\mathrm{p}}(\Sigma), 1<\mathrm{p}<\infty$, is integrable if it has a kernel of the form

$$
\mathrm{K}\left(z, z^{\prime}\right)=\frac{\sum_{i=1}^{n} f_{i}(z) g_{i}\left(z^{\prime}\right)}{z-z^{\prime}}, \quad z, z^{\prime} \in \Sigma ; \quad z \neq z^{\prime}
$$

for such $L^{\infty}$ functions $f_{i}, g_{j}$,

$$
(K h)(z)=\int_{\Sigma} K\left(z, z^{\prime}\right) h\left(z^{\prime}\right) d z^{\prime} .
$$


Integrable operators were first singled out as a distinguished class of operators by Sakhnovich [31] in the late 1960's, and their theory was developed fully by Its, Izergin, Korepin and Slavnov [26] in the early 1990's (see [11] for a full discussion). The famous sine kernel of random matrix theory

$$
\mathrm{K}_{x}\left(z, z^{\prime}\right)=\frac{\sin x\left(z-z^{\prime}\right)}{\pi\left(z-z^{\prime}\right)}=\frac{e^{i x z} e^{-i x z^{\prime}}+\left(-e^{i x z^{\prime}}\right) \cdot e^{i x z}}{2 i \pi\left(z-z^{\prime}\right)}
$$

is a prime example of such an operator, as is likewise the well-known Airy kernel operator.

Integrable operators form an algebra, but their most remarkable property is that their inverses can be expressed in terms of the solution of a naturally associated RHP. Indeed, let $m(z)$ be the solution of the normalized RHP $(\Sigma, v)$ where

$$
v(z)=I-2 \pi i f g^{\top}, \quad f=\left(f_{1}, \ldots, f_{n}\right)^{\top}, g=\left(g_{1}, \ldots, g_{n}\right)^{\top} .
$$

(Here we assume for simplicity that $\sum_{i=1}^{n} f_{i}(z) g_{i}(z)=0$, for all $z \in \Sigma$ as in the sine-kernel: otherwise (4.16) must be slightly modified).

Then $(1-K)^{-1}$ has the form $1+\mathrm{L}$ where $\mathrm{L}$ is an integrable operator

$$
\mathrm{L}\left(z, z^{\prime}\right)=\frac{\sum_{i=1}^{n} F_{i}(z) G_{i}\left(z^{\prime}\right)}{z-z^{\prime}}, \quad z, z^{\prime} \in \Sigma, \quad z \neq z^{\prime}
$$

and

$$
\left\{\begin{array}{l}
F=\left(F_{1}, \ldots, F_{n}\right)^{\top}=m_{ \pm} f \\
G=\left(G_{1}, \ldots, G_{n}\right)^{\top}=\left(m_{ \pm}^{-1}\right)^{\top} g .
\end{array}\right.
$$

This means that if, for example, $\mathrm{K}$ depends on parameters, as in the case of the sine kernel, asymptotic problems involving $\mathrm{K}$ as the parameters become large, are converted into asymptotic problems for a RHP, to which the nonlinear steepest descent method can be applied.

As an example, we show how to use RHP methods to give a proof of Szegö's celebrated Strong Limit Theorem. Let $\mathbb{T}$ be the unit circle.

Theorem 4.18 (Szegő Strong Limit Theorem). Let $\varphi(z)=e^{\mathrm{L}(z)} \in \mathrm{L}^{1}(\mathbb{T}), \varphi(z)>0$, where $\sum_{k=1}^{\infty} k\left|L_{k}\right|^{2}<\infty$ and $\left\{L_{k}\right\}$ are Fourier coefficients of $L(z)$. Let $D_{n}$ be the Toeplitz determinant generated by $\varphi, \mathrm{D}_{n}(\varphi)=\operatorname{det} X(\varphi)$ where $X(\varphi)$ is the $(n+1) \times(n+1)$ matrix with entries $\left\{\varphi_{i-j}\right\}_{0 \leqslant i, j \leqslant n}$, and $\left\{\varphi_{k}\right\}$ are the Fourier coefficients of $\varphi$. Then as $\mathrm{n} \rightarrow \infty$,

$$
D_{n}=e^{(n+1) L_{0}+\sum_{k=1}^{\infty} k\left|L_{k}\right|^{2}}(1+o(1)) .
$$

Sketch of proof. Let $e_{k}, 0 \leqslant k \leqslant n$, be the standard basis in $\mathbb{C}^{n+1}$. Then the map $\mathrm{U}_{\mathrm{n}}: e_{\mathrm{k}} \rightarrow z^{\mathrm{k}}, 0 \leqslant \mathrm{k} \leqslant \mathrm{n}, z \in \mathbb{T}$ takes $\mathbb{C}^{\mathrm{n}+1}$ onto the trigonometric polynomials $\mathcal{P}_{n}=\left\{\sum_{j=0}^{n} a_{j} z^{j}\right\}$ of degree $n$ and induces a map

$$
\tau_{\mathrm{n}}: \mathcal{P}_{\mathrm{n}} \rightarrow \mathcal{P}_{\mathrm{n}}
$$

which is conjugate to $X(\varphi)$. 
We then calculate

$$
\begin{aligned}
\tau_{n} z^{k} & =u_{n} \times u_{n}^{-1} z^{k} \\
& =u_{n} \times e_{k} \\
& =u_{n}\left(\sum_{j=0}^{n} \varphi_{j-k} e_{j}\right) \\
& =\sum_{j=0}^{n} \varphi_{j-k} z^{j}, \quad 0 \leqslant k \leqslant n .
\end{aligned}
$$

Now for any $p=\sum_{k=0}^{n} a_{k} z^{k} \in \mathcal{P}_{n}$

$$
\begin{aligned}
\left(\tau_{\mathrm{n}} p\right)(z) & =\sum_{k=0}^{n} a_{k} \sum_{j=0}^{n} \varphi_{j-k} z^{j} \\
& =\sum_{k=0}^{n} a_{k} \sum_{j=0}^{n}\left(\int_{\Gamma}\left(z^{\prime}\right)^{k-j-1} \varphi\left(z^{\prime}\right) \mathrm{d} z^{\prime}\right) z^{j} \\
& =\sum_{k=0}^{n} a_{k} \int_{\Gamma}\left(z^{\prime}\right)^{k-1} \varphi\left(z^{\prime}\right) \frac{\left(z / z^{\prime}\right)^{n+1}-1}{\left(z / z^{\prime}\right)-1} d z^{\prime} \\
& =\int_{\Gamma} \varphi\left(z^{\prime}\right) p\left(z^{\prime}\right) \frac{\left(z / z^{\prime}\right)^{n+1}-1}{\left(z-z^{\prime}\right)} d z^{\prime} .
\end{aligned}
$$

After some simple calculations (Exercise) one finds that

$$
\tau_{n} p=\left(1-K_{n}\right) p, \quad p \in \mathcal{P}_{n}
$$

where $K_{n}$ is the integrable operator on $\mathbb{T}$ with kernel of the form

$$
K_{n}\left(z, z^{\prime}\right)=\frac{f_{1}(z) g_{1}\left(z^{\prime}\right)+f_{2}(z) g_{2}\left(z^{\prime}\right)}{z-z^{\prime}}, \quad z, z^{\prime} \in \Gamma
$$

where

$$
\begin{aligned}
& f=\left(f_{1}, f_{2}\right)^{\top}=\left(z^{n+1}, 1\right)^{\top} \\
& g=\left(g_{1}, g_{2}\right)^{\top}=\left(z^{-n-1} \frac{1-\varphi(z)}{2 \pi i},-\frac{(1-\varphi(z))}{2 \pi i}\right)^{\top} .
\end{aligned}
$$

We have, in particular, from (4.19) and (4.20), for $0 \leqslant k \leqslant n$,

$$
\left(1-K_{n}\right) z^{k}=\sum_{j=0}^{n} \varphi_{j-k} z^{j}
$$

and for $k<0$ and $k>n$ one easily shows that

$$
\left(1-K_{n}\right) z^{k}=z^{k}+\sum_{j=0}^{n} \varphi_{j-k} z^{i} .
$$

Thus $K_{n}$ is finite rank, and hence trace class, and $\left(1-K_{n}\right)$ has block form with respect to the orthonormal basis $\left\{z^{k}\right\}_{-\infty}^{\infty}$ for $L^{2}(\Gamma)$ as given in Figure 4.23. And so

$$
D_{n}=\operatorname{det} \tau_{n}=\operatorname{det} X(\varphi)=\operatorname{det}\left(1-K_{n}\right)
$$




\begin{tabular}{c|c|c}
$\mathrm{I}$ & 0 & 0 \\
\hline$\cdots$ & $\tau_{\mathrm{n}}$ & $\cdots$ \\
\hline 0 & 0 & $\mathrm{I}$
\end{tabular}

Figure 4.23. The block structure of $1-K_{n}$ in the basis $\left\{z^{k}\right\}_{-\infty}^{\infty}$.

Associated with the integrable operator $K_{n}$ we have the normalized RHP $(\Sigma=\Gamma, v)$ where, by $(4.16),(4.22)$

$$
v=\mathrm{I}-2 \pi \mathrm{ifg} \mathrm{g}^{\top}=\left(\begin{array}{cc}
\varphi & -(\varphi-1) z^{\mathrm{n}+1} \\
z^{-n-1}(\varphi-1) & 2-\varphi
\end{array}\right)
$$

on $\mathbb{T}$. Now

$$
\begin{aligned}
\log D_{n} & =\log \operatorname{det}\left(1-K_{n}\right) \\
& =\operatorname{tr} \log \left(1-K_{n}\right) \\
& =\int_{0}^{1} \frac{d}{d t} \operatorname{tr} \log \left(1-t K_{n}\right) d t \\
& =-\int_{0}^{1} \operatorname{tr}\left(\frac{1}{1-t K_{n}} K_{n}\right) d t .
\end{aligned}
$$

For $0 \leqslant t \leqslant 1$, set

$$
\varphi_{\mathrm{t}}(z)=(1-\mathrm{t})+\mathrm{t} \varphi(z), \quad z \in \mathbb{T} .
$$

Clearly $\varphi_{\mathrm{t}}(z)>0 \quad$ and $\quad \varphi_{0}(z)=1, \quad \varphi_{1}(z)=\varphi(z)$. Now $\quad \varphi_{\mathrm{t}}-1=\mathrm{t}(\varphi-1)$ and so we have from (4.21)

$$
\mathrm{tK}_{\mathrm{n}}=\mathrm{K}_{\mathrm{t}, \mathrm{n}}=\left[\left(\left(z / z^{\prime}\right)^{\mathrm{n}+1}-1\right) /\left(z-z^{\prime}\right)\right]\left[\left(1-\varphi_{\mathrm{t}}\left(z^{\prime}\right)\right) / 2 \pi \mathrm{i}\right]
$$

and it follows that in (4.25)

$$
\begin{aligned}
\frac{1}{1-t K_{n}} t K_{n} & =\frac{1}{1-K_{t, n}} K_{t, n} \\
& =\frac{1}{1-K_{t, n}}-1 \\
& =R_{t, n}
\end{aligned}
$$

where

where by (4.17)

$$
R_{t, n}\left(z, z^{\prime}\right)=\frac{\sum_{j=1}^{2} F_{t, j}(z) G_{t, j}\left(z^{\prime}\right)}{z-z^{\prime}}
$$

$$
\left\{\begin{array}{l}
F_{t}=\left(F_{t, 1}, F_{t, 2}\right)^{\top}=m_{t \pm} f_{t}, \\
G_{t}=\left(G_{t, 1}, G_{t, 2}\right)^{\top}=\left(m_{t \pm}^{-1}\right)^{\top} g_{t} .
\end{array}\right.
$$

Here $m_{t \pm}$ refers to the solution of the RHP $\left(\mathbb{T}, v_{t}\right)$ where $v_{t}$ involves $\varphi_{t}$ rather than $\varphi$ in (4.24), and similarly for $f_{t}, g_{t}$. 
Hence (Exercise)

$$
\log D_{n}=-\int_{0}^{1}\left(\int_{\mathbb{T}}\left(\sum_{j=1}^{2} F_{t, j}^{\prime}(z) G_{t, j}(z)\right) d z\right) \frac{d t}{t} .
$$

So we see that in order to evaluate $D_{n}$ as $n \rightarrow \infty$ we must evaluate the asymptotics of the solution $m_{t}$ of the normalized RHP $\left(\mathbb{T}, v_{t}\right)$ as $n \rightarrow \infty$, for each $0 \leqslant t \leqslant 1$, and substitute this information into (4.27) using (4.26). This is precisely what can be accomplished [11] using the nonlinear steepest descent method.

Here we present the nonlinear steepest descent analysis in the case when $\varphi(z)$ is analytic in an annulus

$$
A_{\epsilon}=\{z: 1-\epsilon<|z|<1+\epsilon\}, \quad \epsilon>0
$$

around $\mathbb{T}$. The idea of the proof, which is a common feature of all applications of the nonlinear steepest descent method, is to move the $z^{n+1}$ term (or its analog in the general situation) in $v_{t}$ into $|z|<1$ and the $z^{-n-1}$ term into $|z|>1$ : then as $n \rightarrow \infty$, these terms are exponentially small, and can be neglected.

But first we must separate the $z^{n+1}$ and $z^{-n-1}$ terms of $v_{\mathrm{t}}$ algebraically. This is done using the lower-upper pointwise factorization of $v_{t}$

$$
v_{\mathrm{t}}=\left(\begin{array}{cc}
1 & 0 \\
z^{-n-1}\left(1-\varphi_{\mathrm{t}}^{-1}\right) & 1
\end{array}\right)\left(\begin{array}{cc}
\varphi_{\mathrm{t}} & 0 \\
0 & \varphi_{\mathrm{t}}^{-1}
\end{array}\right)\left(\begin{array}{cc}
1 & -\left(1-\varphi_{\mathrm{t}}^{-1}\right) z^{\mathrm{n}+1} \\
0 & 1
\end{array}\right)
$$

which is easily verified.

Extend $\mathbb{T}=\Sigma \rightarrow \tilde{\Sigma}=\{|z|=\rho\} \cup \Sigma \cup\left\{|z|=\rho^{-1}\right\}=\Sigma_{\rho} \cup \Sigma \cup \Sigma_{\rho-1}$ where we choose $1-\epsilon<\rho<1<\rho^{-1}<1+\epsilon$. Now define a piecewise analytic function $\tilde{m}$ by the definitions in Figure 4.29.

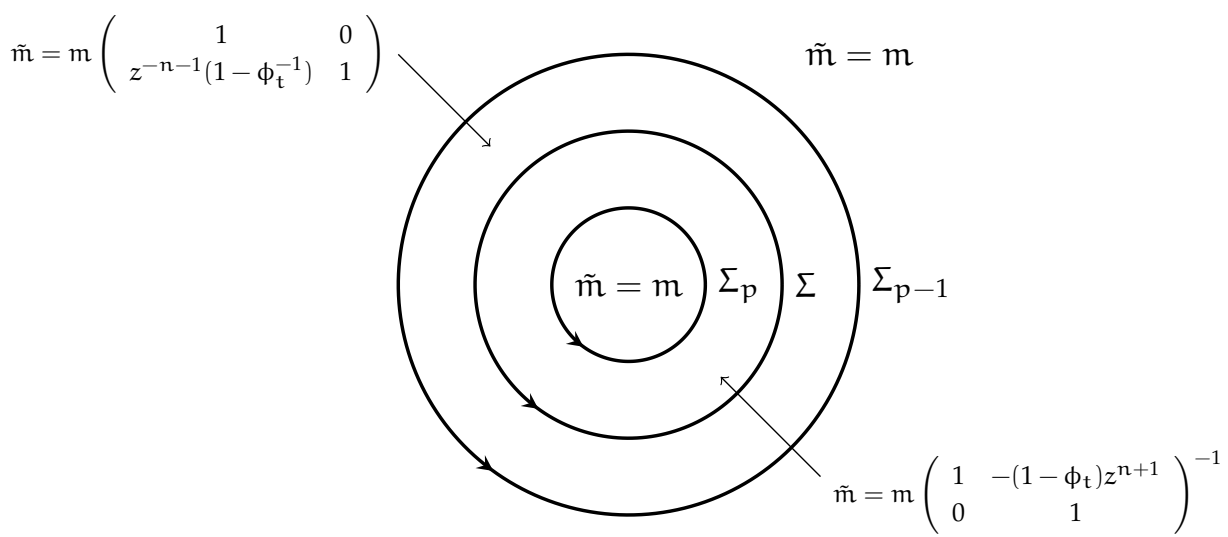

FigURE 4.29. A piecewise definition of $\tilde{m}$.

This definition is motivated by the fact that

$$
\mathrm{m}_{+}=\mathrm{m}_{-} v_{\mathrm{t}}=\mathrm{m}_{-}(\cdot)(\cdot)(\cdot)
$$


as in (4.28). It follows that $\tilde{\mathfrak{m}}(z)$ solves the normalized $\operatorname{RHP}(\tilde{\Sigma}, \tilde{v})$ where

$$
\begin{aligned}
& \tilde{v}(z)=\left(\begin{array}{cc}
1 & 0 \\
z^{-n-1}\left(1-\varphi_{\mathrm{t}}^{-1}\right) & 1
\end{array}\right) \quad \text { on } \quad \Sigma_{\rho^{-1}}, \\
& \tilde{v}(z)=\left(\begin{array}{cc}
\varphi_{\mathfrak{t}}(z) & 0 \\
0 & \varphi_{\mathrm{t}}(z)^{-1}
\end{array}\right) \quad \text { on } \quad \Sigma, \\
& \tilde{v}(z)=\left(\begin{array}{cc}
1 & -\left(1-\varphi_{\mathrm{t}}^{-1}\right) z^{\mathrm{n}+1} \\
0 & 1
\end{array}\right) \quad \text { on } \quad \Sigma_{\rho} .
\end{aligned}
$$

Now as $n \rightarrow \infty, \tilde{v}(z) \rightarrow$ I on $\Sigma_{\rho}$ and on $\Sigma_{\rho-1}$. This means that $\tilde{m} \rightarrow m_{\infty}$ where $\mathrm{m}_{\infty}$ solves the normalized RHP $\left(\Sigma, v_{\infty}\right)$ where

$$
v_{\infty}=\left.v\right|_{\Sigma}=\left(\begin{array}{cc}
\varphi_{\mathrm{t}} & 0 \\
0 & \varphi_{\mathrm{t}}^{-1}
\end{array}\right) .
$$

But this RHP is a direct sum of scalar RHP's and hence can be solved explicitly, as noted earlier (cf. (3.31)). In this way we obtain the asymptotics of $m$ as $n \rightarrow \infty$ and hence the asymptotics of the Toeplitz determinant $D_{n}$.

Here is what, alas, I have not done and what I had hoped to do in these lectures (see AMS open notes):

- Show that in addition to the usefulness of RHP's for algebraic and asymptotic purposes, RHP's are also useful for analytic purposes. In particular, RHP's can be used to show that the Painlevé equations indeed have the Painlevé property.

- Show that in addition to RHP's arising "out of the blue" as in the case of orthogonal polynomials and systematically in the case of ODE's and also integrable operators, RHP's also arise in a systematic fashion in WienerHopf Theory.

- Describe what happens to an RHP when the operator $1-\mathrm{C}_{\omega}$ is Fredholm, but not bijective, and

- Finally, I have not succeeded in showing you how the nonlinear steepest descent method works in general. All I have shown is one simple case.

\section{References}

[1] M Abramowitz and I A Stegun, Handbook of mathematical functions, National Bureau of Standards, Washington D.C., 1970. $\leftarrow 1,2,3$

[2] J Baik, P Deift, and K Johansson, On the distribution of the length of the longest increasing subsequence of random permutations, J. Am. Math. Soc. 12 (1999oct), no. 04, 1119-1179. $\leftarrow 4$

[3] J Baik, P Deift, and Suidan, Combinatorics and Random Matrix Theory, Amer. Math. Soc., Providence, RI, 2017. $\leftarrow 5$

[4] R Beals and R R Coifman, Scattering and inverse scattering for first order systems, Comm. Pure Appl. Math. 37 (1984), 39-90. $\leftarrow 33$

[5] R Beals, P Deift, and C Tomei, Direct and inverse scattering on the line, Mathematical Surveys and Monographs, vol. 28, American Mathematical Society, Providence, RI, 1988. $\leftarrow 12,16$ 
[6] A Böttcher and Y I Karlovich, Carleson Curves, Muckenhoupt Weights, and Toeplitz Operators, Progress in Mathematics, vol. 154, Birkhäuser Verlag, Basel, 1997. $\leftarrow 12,19,20$

[7] A P Calderón, Cauchy integrals on Lipschitz curves and related operators, Proc. Nat. Acad. Sci. 74 (1977), 1324-1327. $\leftarrow 19$

[8] K F Clancey and I Gohberg, Factorization of matrix functions and singular integral operators, Operator Theory: Advances and Applications, vol. 3, Birkhäuser Verlag, Basel, 1981. $\leftarrow 1,12,20$

[9] R R Coifman, A McIntosh, and Y Meyer, L'integrale de Cauchy définit un opérateur borné sur L ${ }^{2}$ pour les courbes Lipschitziennes, Ann. of Math. 116 (1982), $361-388 . \leftarrow 19$

[10] G David, L'integrale de Cauchy sur les courbes rectifiables, Prepublication Univ. Paris-Sud, Dept. Math. 82T05 (1982). $\leftarrow 19$

[11] P Deift, Integrable operators, Amer. Math. Soc. Transl. 198 (1999), no. 2, 69-84. $\leftarrow 35,38$

[12] P Deift, Orthogonal Polynomials and Random Matrices: a Riemann-Hilbert Approach, Amer. Math. Soc., Providence, RI, 2000. $\leftarrow 1,31$

[13] P Deift and D Gioev, Random Matrix Theory: Invariant Ensembles and Universality, Courand Lecture Notes, vol. 18, Amer. Math. Soc., Providence, RI, 2009. $\leftarrow 31$

[14] P Deift, T Kriecherbauer, K T-R McLaughlin, S Venakides, and X Zhou, Asymptotics for polynomials orthogonal with respect to varying exponential weights, Internat. Math. Res. Not. 16 (1997), 759-782. $\leftarrow 31$

[15] P Deift, T Kriecherbauer, K T-R McLaughlin, S Venakides, and X Zhou, Uniform asymptotics for polynomials orthogonal with respect to varying exponential weights and applications to universality questions in random matrix theory, Commun. Pure Appl. Math. 52 (1999nov), no. 11, 1335-1425. $\leftarrow 31$

[16] P Deift and X Zhou, A steepest descent method for oscillatory Riemann-Hilbert problems. Asymptotics for the MKdV Equation, Ann. Math. 137 (1993), no. 2, 295-368. $\leftarrow 4$, 5, 7, 9

[17] P Deift and X Zhou, Long-time asymptotics for solutions of the NLS equation with initial data in a weighted Sobolev space, Comm. Pure Appl. Math. 56 (2003aug), 1029-1077, available at 0206222v2. $\leftarrow 7,20,32$

[18] P Deift and X Zhou, Asymptotics for the painlevé II equation, Commun. Pure Appl. Math. 48 (1995), no. 3, 277-337. $\leftarrow 7,8$

[19] P Duren, Theory of $\mathrm{H}^{\mathrm{p}}$ Spaces, Academic Press, 1970. $\leftarrow 1,10$

[20] H Flaschka and A C Newell, Monodromy and spectrum preserving deformations, I, Comm. Math. Phys. 76, 67-116. $\leftarrow 7$

[21] A S Fokas, A R Its, and A V Kitaev, The isomonodromy approach to matrix models in $2 D$ quantum gravity, Commun. Math. Phys. 147 (1992), 395-430. $\leftarrow 31$

[22] A S Fokas, A R Its, A A Kapaev, and V Y Novokshenov, Painlevé Transcendents: the RiemannHilbert Approach, Amer. Math. Soc., 2006. $\leftarrow 6,8,29$

[23] J Garnett, Bounded Analytic Functions, Graduate Texts in Mathematics, Springer New York, New York, NY, 2007. $\leftarrow 1,10$

[24] G M Goluzin, Geometric Theory of Functions of a Complex Variable, Amer. Math. Soc., Providence, RI, 1969. $\leftarrow 1,18$

[25] A R Its, A S Fokas, and A A Kapaev, On the asymptotic analysis of the Painleve equations via the isomonodromy method, Nonlinearity 7 (1994sep), no. 5, 1291-1325. $\leftarrow 8$

[26] A R Its, V E Izergin, V E Korepin, and N A Slavnov, Differential equations for quantum correlation functions, Int. J. Mod. Phys. B 4 (1990), 1003. $\leftarrow 35$

[27] M Jimbo and T Miwa, Monodromy preserving deformations of linear ordinary differential equation with rational coefficients: II., Physica D 2 (1981), 407-448. $\leftarrow 7$

[28] G S Litvinchuk and I M Spitkovskii, Factorization of Measurable Matrix Functions, Operator Theory: Advances and Applications, vol. 25, Birkhäuser Basel, Basel, 1987. $\leftarrow 1,20$

[29] F W J Olver, D W Lozier, R F Boisvert, and C W Clark, NIST Handbook of Mathematical Functions, Cambridge University Press, 2010. $\leftarrow 1$

[30] H Royden and P Fitzpatrick, Real analysis, 4th ed., Pearson, London, 2010. $\leftarrow 17$

[31] L A Sakhnovich, Operators similar to the unitary operator with absolutely continuous spectrum, Functional Anal. and Appl. 2 (1968), 48-60. $\leftarrow 35$

Department of Mathematics, Courant Institute of Mathematical Sciences, New York University E-mail address: deift@cims.nyu.edu 